\title{
A 14-dimensional module for the symplectic group: orbits on vectors
}

\author{
B. De Bruyn and M. Kwiatkowski \\ Ghent University, Department of Mathematics, Krijgslaan 281 (S22), B-9000 Gent, Belgium, \\ E-mail: bdb@cage.ugent.be/mkw@cage.ugent.be
}

\begin{abstract}
Let $\mathbb{F}$ be a field, $V$ a 6 -dimensional $\mathbb{F}$-vector space and $f$ a nondegenerate alternating bilinear form on $V$. We consider a 14-dimensional module for the symplectic group $S p(V, f) \cong S p(6, \mathbb{F})$ associated with $(V, f)$, and classify the orbits on vectors. For characteristic distinct from 2, this module is irreducible and isomorphic to the Weyl module of $S p(V, f)$ for the fundamental weight $\lambda_{3}$. If the characteristic is 2 , then the module is reducible as it contains an 8-dimensional submodule isomorphic to the spin module of $S p(V, f)$.
\end{abstract}

Keywords: symplectic group, Weyl module, spin module, exterior power, trivector, (quasi) $S p(V, f)$-equivalence

MSC2000: $15 \mathrm{~A} 75,15 \mathrm{~A} 63,20 \mathrm{C}$

\section{Introduction}

Let $\mathbb{F}$ be a field and $V$ a 6 -dimensional $\mathbb{F}$-vector space equipped with a nondegenerate alternating bilinear form $f$. The symplectic group $G=S p(V, f) \cong S p(6, \mathbb{F})$ associated with the symplectic space $(V, f)$ has a natural action on the third exterior power $\bigwedge^{3} V$ of $V$. The corresponding 20-dimensional $\mathbb{F} G$-module has two nontrivial submodules, one of dimension 14 which we will denote by $W$ and another one of dimension 6 which we will denote by $\widetilde{W}$. The 14-dimensional submodule $W$ is generated by all decomposable trivectors of the form $v_{1} \wedge v_{2} \wedge v_{3}$, where $\left\langle v_{1}, v_{2}, v_{3}\right\rangle$ is a 3 -space totally isotropic for $f$. This module is the Weyl module of $S p(V, f)$ for the fundamental weight $\lambda_{3}$, see Premet and Suprunenko [27]. The 6-dimensional submodule $\widetilde{W}$ consists of all trivectors $\alpha \in \bigwedge^{3} V$ such that $\alpha \wedge \beta=0$ for all $\beta \in W$. The module $\widetilde{W}$ is isomorphic to $V$, regarded (in a natural way) as an $\mathbb{F} G$-module.

For characteristic 0, Maschke's theorem guarantees that the $\mathbb{F} G$-module $\bigwedge^{3} V$ can be written as the direct sum of irreducible submodules. In fact, this property holds as soon as the characteristic of $\mathbb{F}$ is distinct from 2. Indeed, in case the characteristic is distinct 
from 2 , the submodules $W$ and $\widetilde{W}$ are irreducible and $\bigwedge^{3} V=W \oplus \widetilde{W}$. On the other hand, if $\operatorname{char}(\mathbb{F})=2$, then $\widetilde{W} \subseteq W$ and so $W$ cannot be irreducible. Besides $W$ and $\widetilde{W}$, we can also consider the $\mathbb{F} G$-modules on the quotient spaces $\wedge^{3} V / W$ and $\bigwedge^{3} V / \widetilde{W}$. The first module is isomorphic to $V$, and latter module is isomorphic to $W$ if the characteristic is distinct from 2. If the characteristic equals 2, then the submodule $\wedge^{3} V / \widetilde{W}$ is reducible as it contains $\{w+\widetilde{W} \mid w \in W\}$ as an 8-dimensional submodule. This submodule is isomorphic to the spin module for $S p(V, f)$, see Gow [19].

The aim of this paper is to classify the orbits on vectors of the $\mathbb{F} G$-module $\wedge^{3} V / \widetilde{W}$. The difficulty of the problem and the methods to solve it heavily depend on the characteristic of $\mathbb{F}$. The case where $\operatorname{char}(\mathbb{F}) \neq 2$ is the easiest one. In this case, the module is isomorphic to $W$ and the orbits on vectors of $W$ were already described in the literature. For algebraically closed fields of characteristic distinct from 2, these orbits were determined by Igusa [24, p. 1027]. For general fields, these orbits can be extracted from a series of four papers $[13,14,15,16]$ by the authors, where they succeeded in obtaining a complete classification of all orbits on vectors of the $S p(V, f)$-module $\wedge^{3} V$, hereby extending a result of Popov [26] who succeeded in the same goal, but under the extra assumption that the underlying field $\mathbb{F}$ is algebraically closed of characteristic distinct from 2. The results of the papers $[13,14,15,16]$, which will be recalled in Section 2, will play an important role the present paper to obtain the desired classification results in the characteristic 2 case.

The problem of classifying orbits on vectors (or on subspaces) of certain group modules has already been considered before in the literature. All finite-dimensional irreducible rational $\mathbb{K} H$-modules on which a group $H$ has a finite number of orbits on vectors have been determined in Guralnick et al. [20] in case $H$ is a connected linear algebraic group over an algebraically closed field $\mathbb{K}$. For the purpose of studying the subgroup structure of the Chevalley groups of type $E_{6}$, Aschbacher studied the 27-dimensional modules for these groups. In particular, he classified the orbits on vectors and hyperplanes of these modules, see [1]. Cooperstein [7] classified orbits on vectors of the 57-dimensional modules for the Chevalley groups of type $E_{7}$, also with the intention to use this information to study the subgroup structure. There are a number of other papers dealing with the problem of classifying orbits on vectors and subspaces of certain group modules, see e.g. [3, 5]. For group modules involving a general linear group $G L(V)$ acting on an exterior power of $V$, we also have a number of results dealing with the classification of orbits on vectors, see $[2,4,18,21,22,23,28,29,30,32,33,34]$. Some of these results however impose certain restrictions on the underlying field.

One of the motivations for studying the problem under consideration in this paper is the so-called isomorphism problem for hyperplanes of symplectic dual polar spaces. With the pair $(V, f)$, there is associated a symplectic dual polar space $D W(5, \mathbb{F})$. This is the point-line geometry whose points are the 3-spaces of $V$ totally isotropic for $f$, with each line being the collection of all totally isotropic 3-spaces that contain a given totally isotropic 2-space. A hyperplane of a point-line geometry is a set of points, distinct from the whole point-set, meeting each line in either one or all of its points. If the point-line 
geometry is fully embeddable in a projective space, then there is a standard way of constructing hyperplanes, namely by intersecting the embedded geometry with hyperplanes of the ambient projective space. Hyperplanes that arise in this way are called classical. If the field $\mathbb{F}$ has at least three elements, then theoretical results of Cooperstein [8], Kasikova and Shult [25] and Ronan [31] imply that all classical hyperplanes of $D W(5, \mathbb{F})$ must arise from the so-called Grassmann embedding of $D W(5, \mathbb{F})$. Without going into technical details, this amounts to saying that if $|\mathbb{F}| \geq 3$, then there exists some one-toone correspondence between the classical hyperplanes of $D W(5, \mathbb{F})$ and the 1-spaces of the quotient module $\bigwedge^{3} V / \widetilde{W}$ under consideration in the present paper. The knowledge of the orbits of vectors of this quotient module seems indispensable to obtain a classification of the isomorphism classes of hyperplanes of $D W(5, \mathbb{F})$. Such classifications for hyperplanes have already been obtained in the case the underlying field is perfect of characteristic 2 (De Bruyn [10]) or finite and of odd characteristic (Cooperstein and De Bruyn [9]). The results of the present paper will allow to generalize some of the results contained in these papers.

The group $G L(V)$ has a natural action on $\bigwedge^{3} V$. Indeed, for every $\theta \in G L(V)$, there exists a unique $\bigwedge^{3}(\theta) \in G L\left(\bigwedge^{3} V\right)$ such that $\bigwedge^{3}(\theta)\left(v_{1} \wedge v_{2} \wedge v_{3}\right)=\theta\left(v_{1}\right) \wedge \theta\left(v_{2}\right) \wedge \theta\left(v_{3}\right)$ for all $v_{1}, v_{2}, v_{3} \in V$. In the sequel, we will often write $\theta$ instead of $\Lambda^{3}(\theta)$, accepting this abuse of notation for the gain of readability. Using this notation, we say that two trivectors $\chi_{1}$ and $\chi_{2}$ are $G$-equivalent for some subgroup $G$ of $G L(V)$ if $\chi_{2}=\theta\left(\chi_{1}\right)$ for some $\theta \in G$. We can define an equivalence relation on the vectors of $\bigwedge^{3} V$ which is coarser than $S p(V, f)$ equivalence. We say that two trivectors $\chi_{1}$ and $\chi_{2}$ are quasi-Sp $(V, f)$-equivalent if there exists a $\theta \in S p(V, f)$ and a $\chi \in \widetilde{W}$ such that $\chi_{2}=\theta\left(\chi_{1}\right)+\chi$. Obviously, there is a bijective correspondence between the quasi- $S p(V, f)$-equivalence classes and the orbits on vectors of the $\mathbb{F} G$-module $\bigwedge^{3} V / \widetilde{W}$. In view of this, we prefer to state our main results in terms of this quasi- $S p(V, f)$-equivalence relation. Before we can do that, we still need to discuss a result of Revoy regarding the classification of the $G L(V)$-equivalence classes of trivectors of $V$.

Put $\mathbb{F}^{*}:=\mathbb{F} \backslash\{0\}$ and let $\overline{\mathbb{F}}$ be a fixed algebraic closure of $\mathbb{F}$. For every separable quadratic extension $\mathbb{F}^{\prime}$ of $\mathbb{F}$ contained in $\overline{\mathbb{F}}$, we choose one pair $\left(a_{\mathbb{F}^{\prime}}, b_{\mathbb{F}^{\prime}}\right) \in \mathbb{F}^{2}$ such that the quadratic polynomial $X^{2}-a_{\mathbb{F}^{\prime}} X-b_{\mathbb{F}^{\prime}} \in \mathbb{F}[X]$ is irreducible and $\mathbb{F}^{\prime} \subseteq \overline{\mathbb{F}}$ is the quadratic extension of $\mathbb{F}$ defined by this polynomial. In general, there are many possibilities for $\left(a_{\mathbb{F}^{\prime}}, b_{\mathbb{F}^{\prime}}\right)$, but throughout this paper $\left(a_{\mathbb{F}^{\prime}}, b_{\mathbb{F}^{\prime}}\right)$ will be a fixed choice among all these possibilities. For every nonseparable quadratic extension $\mathbb{F}^{\prime} \subseteq \overline{\mathbb{F}}$ of $\mathbb{F}$, we put $a_{\mathbb{F}^{\prime}}:=0$ and we choose a nonsquare $b_{\mathbb{F}^{\prime}}$ in $\mathbb{F}$ such that $\mathbb{F}^{\prime} \subseteq \overline{\mathbb{F}}$ is the quadratic extension of $\mathbb{F}$ defined by the irreducible quadratic polynomial $X^{2}+b_{\mathbb{F}^{\prime}} \in \mathbb{F}[X]$. There are many possibilities for $b_{\mathbb{F}^{\prime}}$, but throughout this paper $b_{\mathbb{F}^{\prime}}$ will be a fixed choice among all these possibilities. Put

$$
\begin{aligned}
\Psi & :=\left\{\left(a_{\mathbb{F}^{\prime}}, b_{\mathbb{F}^{\prime}}\right) \mid \mathbb{F}^{\prime} \subseteq \overline{\mathbb{F}} \text { is a separable quadratic extension of } \mathbb{F}\right\} \\
\Psi^{\prime} & :=\left\{b_{\mathbb{F}^{\prime}} \mid \mathbb{F}^{\prime} \subseteq \overline{\mathbb{F}} \text { is a nonseparable quadratic extension of } \mathbb{F}\right\} .
\end{aligned}
$$

Let $\left\{v_{1}, v_{2}, v_{3}, v_{4}, v_{5}, v_{6}\right\}$ be a fixed basis of $V$. For every quadratic extension $\mathbb{F}^{\prime}$ of $\mathbb{F}$ 
contained in $\overline{\mathbb{F}}$, we have $b_{\mathbb{F}^{\prime}} \neq 0$ and we define

$$
\chi_{\mathbb{F}^{\prime}}^{*}:=\lambda_{\mathbb{F}^{\prime}} \cdot v_{1} \wedge v_{2} \wedge v_{3}+\mu_{\mathbb{F}^{\prime}} \cdot v_{4} \wedge v_{5} \wedge v_{6}+\left(v_{1}+v_{4}\right) \wedge\left(v_{2}+v_{5}\right) \wedge\left(v_{3}+v_{6}\right),
$$

where $\lambda_{\mathbb{F}^{\prime}}:=a_{\mathbb{F}^{\prime}}+b_{\mathbb{F}^{\prime}}-1$ and $\mu_{\mathbb{F}^{\prime}}:=\frac{1-a_{\mathbb{F}^{\prime}}-b_{\mathbb{F}^{\prime}}}{b_{\mathbb{F}^{\prime}}}$.

A complete classification of all $G L(V)$-equivalence classes of trivectors of $V$ was obtained by Revoy [29].

Proposition 1.1 ([29]) Let $\left\{v_{1}, v_{2}, \ldots, v_{6}\right\}$ be the fixed basis of $V$ as above. Then every nonzero trivector of $V$ is $G L(V)$-equivalent with precisely one of the following vectors:

(A) $v_{1} \wedge v_{2} \wedge v_{3}$

(B) $v_{1} \wedge v_{2} \wedge v_{3}+v_{1} \wedge v_{4} \wedge v_{5}$

(C) $v_{1} \wedge v_{2} \wedge v_{3}+v_{4} \wedge v_{5} \wedge v_{6}$

(D) $v_{1} \wedge v_{2} \wedge v_{4}+v_{1} \wedge v_{3} \wedge v_{5}+v_{2} \wedge v_{3} \wedge v_{6}$

(E) $\chi_{\mathbb{F}^{\prime}}^{*}$ for some quadratic extension $\mathbb{F}^{\prime}$ of $\mathbb{F}$ contained in $\overline{\mathbb{F}}$.

If $\mathbb{F}_{1}^{\prime}$ and $\mathbb{F}_{2}^{\prime}$ are two distinct quadratic extensions of $\mathbb{F}$ contained in $\overline{\mathbb{F}}$, then the trivectors $\chi_{\mathbb{F}_{1}^{\prime}}^{*}$ and $\chi_{\mathbb{F}_{2}^{\prime}}^{*}$ are not $G L(V)$-equivalent.

Let $X \in\{A, B, C, D, E\}$. A nonzero trivector of $V$ is said to be of Type (X) if it is $G L(V)$ equivalent with (one of) the trivector(s) described in (X) of Proposition 1.1. It should be mentioned that the description of the trivectors of Type (E) in terms of the parameters $\lambda_{\mathbb{F}^{\prime}}$ and $\mu_{\mathbb{F}^{\prime}}$ is not taken from Revoy's paper [29], but from the paper [12] of one of the authors. A complete classification of all $G L(V)$-equivalence classes of trivectors of $V$ was also obtained by a number of other people under certain assumptions of the underlying field $\mathbb{F}$, see for instance Cohen and Helminck [4] and Reichel [28].

The next two theorems give a complete classification of all quasi- $S p(V, f)$-equivalence classes in the case the characteristic of $\mathbb{F}$ is distinct from 2. These two theorems will be proved in Section 2 and are an almost immediate consequence of the classification of the $S p(V, f)$-equivalence classes of trivectors. In the remainder of this introductory section, $\left(e_{1}, f_{1}, e_{2}, f_{2}, e_{3}, f_{3}\right)$ will denote a fixed hyperbolic basis of the symplectic space $(V, f)$, that means that $f\left(e_{i}, e_{j}\right)=f\left(f_{i}, f_{j}\right)=0$ and $f\left(e_{i}, f_{j}\right)=\delta_{i j}$ for all $i, j \in\{1,2,3\}$.

Theorem 1.2 (Section 2) Suppose $\operatorname{char}(\mathbb{F}) \neq 2$. Then every trivector of $V$ is quasi$S p(V, f)$-equivalent with (at least) one of the following trivectors:

(Q1) the zero vector of $\bigwedge^{3} V$;

$(Q 2) \chi_{A 1}=e_{1} \wedge e_{2} \wedge e_{3}$

(Q3) $\chi_{B 4}(\lambda)=e_{1} \wedge e_{2} \wedge e_{3}+\lambda \cdot e_{1} \wedge f_{2} \wedge f_{3}$ for some $\lambda \in \mathbb{F}^{*}$;

(Q4) $\chi_{C 1}(\lambda)=e_{1} \wedge e_{2} \wedge e_{3}+\lambda \cdot f_{1} \wedge f_{2} \wedge f_{3}$ for some $\lambda \in \mathbb{F}^{*}$;

(Q5) $\chi_{D 3}\left(\lambda_{1}, \lambda_{2}\right)=e_{1} \wedge e_{2} \wedge f_{3}+\lambda_{1} \cdot e_{2} \wedge e_{3} \wedge f_{1}+\lambda_{2} \cdot e_{3} \wedge e_{1} \wedge f_{2}$ for some $\lambda_{1}, \lambda_{2} \in \mathbb{F}^{*}$; 
(Q6) $\chi_{E 1}\left(a, b, h_{1}, h_{2}, h_{3}\right)=2 \cdot e_{1} \wedge e_{2} \wedge e_{3}+a \cdot\left(h_{1} \cdot f_{1} \wedge e_{2} \wedge e_{3}+h_{2} \cdot e_{1} \wedge f_{2} \wedge e_{3}+h_{3}\right.$. $\left.e_{1} \wedge e_{2} \wedge f_{3}\right)+\left(a^{2}+2 b\right) \cdot\left(h_{1} h_{2} \cdot f_{1} \wedge f_{2} \wedge e_{3}+h_{1} h_{3} \cdot f_{1} \wedge e_{2} \wedge f_{3}+h_{2} h_{3} \cdot e_{1} \wedge f_{2} \wedge\right.$ $\left.f_{3}\right)+h_{1} h_{2} h_{3} a\left(a^{2}+3 b\right) \cdot f_{1} \wedge f_{2} \wedge f_{3}$ for some $(a, b) \in \Psi$ and some $h_{1}, h_{2}, h_{3} \in \mathbb{F}^{*}$.

In the case $\operatorname{char}(\mathbb{F}) \neq 2$, a trivector of $V$ is said to be of Type $(Q i), i \in\{1,2, \ldots, 6\}$, if it is quasi- $S p(V, f)$-equivalent with (one of) the trivector(s) defined in (Q $i$ ) of Theorem 1.2.

Theorem 1.3 (Section 2) Suppose $\operatorname{char}(\mathbb{F}) \neq 2$.

- Let $i, j \in\{1,2, \ldots, 6\}$ with $i \neq j$. Then no trivector of Type (Qi) is quasi-Sp $(V, f)$ equivalent with a trivector of Type $(Q j)$.

- Let $\lambda, \lambda^{\prime} \in \mathbb{F}^{*}$. Then the two trivectors $\chi_{B 4}(\lambda)$ and $\chi_{B 4}\left(\lambda^{\prime}\right)$ of $V$ are quasi-Sp $(V, f)$ equivalent if and only if $\frac{\lambda}{\lambda^{\prime}}$ is a square in $\mathbb{F}$.

- Let $\lambda, \lambda^{\prime} \in \mathbb{F}^{*}$. Then the two trivectors $\chi_{C 1}(\lambda)$ and $\chi_{C 1}\left(\lambda^{\prime}\right)$ of $V$ are quasi-Sp $(V, f)$ equivalent if and only if $\lambda^{\prime} \in\{\lambda,-\lambda\}$.

- Let $\lambda_{1}, \lambda_{2}, \lambda_{1}^{\prime}, \lambda_{2}^{\prime} \in \mathbb{F}^{*}$. Then the two trivectors $\chi_{D 3}\left(\lambda_{1}, \lambda_{2}\right)$ and $\chi_{D 3}\left(\lambda_{1}^{\prime}, \lambda_{2}^{\prime}\right)$ of $V$ are quasi-Sp $(V, f)$-equivalent if and only if the matrices $\operatorname{diag}\left(\lambda_{1}, \lambda_{2}, \lambda_{1} \lambda_{2}\right)$ and $\operatorname{diag}\left(\lambda_{1}^{\prime}, \lambda_{2}^{\prime}, \lambda_{1}^{\prime} \lambda_{2}^{\prime}\right)$ are congruent, i.e. if and only if there exists a nonsingular $(3 \times 3)$ matrix $A$ over $\mathbb{F}$ such that $\operatorname{diag}\left(\lambda_{1}, \lambda_{2}, \lambda_{1} \lambda_{2}\right)=A \cdot \operatorname{diag}\left(\lambda_{1}^{\prime}, \lambda_{2}^{\prime}, \lambda_{1}^{\prime} \lambda_{2}^{\prime}\right) \cdot A^{T}$.

- Let $h_{1}, h_{2}, h_{3}, h_{1}^{\prime}, h_{2}^{\prime}, h_{3}^{\prime} \in \mathbb{F}^{*}$ and $(a, b),\left(a^{\prime}, b^{\prime}\right) \in \Psi$. Then the two trivectors $\chi_{E 1}(a, b$, $\left.h_{1}, h_{2}, h_{3}\right)$ and $\chi_{E 1}\left(a^{\prime}, b^{\prime}, h_{1}^{\prime}, h_{2}^{\prime}, h_{3}^{\prime}\right)$ of $V$ are quasi-Sp $(V, f)$-equivalent if and only if $(a, b)=\left(a^{\prime}, b^{\prime}\right)$ and there exists a $3 \times 3$-matrix $A$ over $\mathbb{F}^{\prime}$ with determinant equal to 1 such that $A \cdot \operatorname{diag}\left(h_{1}, h_{2}, h_{3}\right) \cdot\left(A^{\psi}\right)^{T}$ is equal to $\operatorname{diag}\left(h_{1}^{\prime}, h_{2}^{\prime}, h_{3}^{\prime}\right)$ or $\operatorname{diag}\left(-h_{1}^{\prime},-h_{2}^{\prime},-h_{3}^{\prime}\right)$. Here, $\mathbb{F}^{\prime} \subseteq \overline{\mathbb{F}}$ is the quadratic extension of $\mathbb{F}$ determined by the irreducible quadratic polynomial $X^{2}-a X-b$ of $\mathbb{F}[X]$ and $\psi$ is the unique nontrivial element of the Galois group $\operatorname{Gal}\left(\mathbb{F}^{\prime} / \mathbb{F}\right)$.

In Theorem 1.3 $\operatorname{diag}\left(h_{1}, h_{2}, h_{3}\right)$ denotes the $(3 \times 3)$-diagonal matrix whose $(i, i)$-th entry is equal to $h_{i}$ for every $i \in\{1,2,3\}$. In the next two theorems, we describe the obtained classification results for the quasi- $S p(V, f)$-equivalence classes in the case the characteristic of the field $\mathbb{F}$ is equal to 2. These two theorems will be proved in Section 4 .

Theorem 1.4 (Section 4) Suppose char $(\mathbb{F})=2$. Let $\chi$ be a trivector of $V$ which is quasi-Sp $(V, f)$-equivalent with a trivector of Type $(A),(B),(C)$ or $(D)$. Then $\chi$ is quasi$S p(V, f)$-equivalent with (at least) one of the following trivectors:

$\left(Q 1^{\prime}\right)$ the zero vector of $\bigwedge^{3} V$;

$\left(Q 2^{\prime}\right) \chi_{A 1}=e_{1} \wedge e_{2} \wedge e_{3} ;$ 
$\left(Q 3^{\prime}\right) \chi_{A 2}=e_{1} \wedge e_{2} \wedge f_{2} ;$

$\left(Q 4^{\prime}\right) \chi_{B 4}(\lambda)=e_{1} \wedge e_{2} \wedge e_{3}+\lambda \cdot e_{1} \wedge f_{2} \wedge f_{3}$ for some nonsquare $\lambda$ of $\mathbb{F} ;$

$\left(Q 5^{\prime}\right) \chi_{B 5}(\lambda)=\lambda \cdot e_{1} \wedge e_{2} \wedge f_{2}+e_{1} \wedge\left(e_{2}-e_{3}\right) \wedge\left(f_{2}+f_{3}\right)$ for some $\lambda \in \mathbb{F}$ such that the polynomial $X^{2}+\lambda X+1 \in \mathbb{F}[X]$ is irreducible;

$\left(Q 6^{\prime}\right) \chi_{C 1}(\lambda)=e_{1} \wedge e_{2} \wedge e_{3}+\lambda \cdot f_{1} \wedge f_{2} \wedge f_{3}$ for some $\lambda \in \mathbb{F}^{*}$

$\left(Q 7^{\prime}\right) \chi_{D 2}(\lambda)=\lambda \cdot e_{1} \wedge e_{2} \wedge f_{3}+e_{2} \wedge f_{1} \wedge e_{3}+f_{1} \wedge e_{1} \wedge f_{2}$ for some $\lambda \in \mathbb{F}^{*}$

$\left(Q 8^{\prime}\right) \chi_{D 3}\left(\lambda_{1}, \lambda_{2}\right)=e_{1} \wedge e_{2} \wedge f_{3}+\lambda_{1} \cdot e_{2} \wedge e_{3} \wedge f_{1}+\lambda_{2} \cdot e_{3} \wedge e_{1} \wedge f_{2}$ for some $\lambda_{1}, \lambda_{2} \in \mathbb{F}^{*}$ such that the equation $\lambda_{1} X^{2}+\lambda_{2} Y^{2}+Z^{2}=0$ has no solutions for $(X, Y, Z) \in \mathbb{F}^{3} \backslash\{(0,0,0)\}$;

$\left(Q 9^{\prime}\right) \chi_{D 4}\left(\lambda_{1}, \lambda_{2}\right)=e_{1} \wedge e_{2} \wedge f_{3}+\lambda_{1} \cdot e_{2} \wedge e_{3} \wedge\left(f_{1}+f_{3}\right)+\lambda_{2} \cdot e_{3} \wedge e_{1} \wedge f_{2}$ for some $\lambda_{1}, \lambda_{2} \in \mathbb{F}^{*}$.

In the case $\operatorname{char}(\mathbb{F})=2$, a trivector of $V$ is said to be of Type $\left(Q i^{\prime}\right), i \in\{1,2, \ldots, 9\}$, if it is quasi- $S p(V, f)$-equivalent with (one of) the trivector(s) defined in (Q $\left.i^{\prime}\right)$ of Theorem 1.4. Two $(3 \times 3)$-matrices $A_{1}$ and $A_{2}$ over $\mathbb{F}$ are called pseudo-congruent if there exists a nonsingular $(3 \times 3)$-matrix $M$ over $\mathbb{F}$ such that the matrix $A_{1}-M A_{2} M^{T}$ is alternating, i.e. skew-symmetric and having all diagonal elements equal to 0 . The relation of being pseudo-congruent defines an equivalence relation of the set of all $(3 \times 3)$-matrices over $\mathbb{F}$.

Theorem 1.5 (Section 4) Suppose $\operatorname{char}(\mathbb{F})=2$.

- Let $i, j \in\{1,2, \ldots, 9\}$ with $i \neq j$. Then no trivector of Type $\left(Q i^{\prime}\right)$ is quasi-Sp $(V, f)$ equivalent with a trivector of Type $\left(Q j^{\prime}\right)$.

- Let $\lambda$ and $\lambda^{\prime}$ be two nonsquares of $\mathbb{F}$. Then the two trivectors $\chi_{B 4}(\lambda)$ and $\chi_{B 4}\left(\lambda^{\prime}\right)$ are quasi-Sp $(V, f)$-equivalent if and only if the polynomials $X^{2}+\lambda$ and $X^{2}+\lambda^{\prime}$ define the same quadratic extension of $\mathbb{F}$ in $\overline{\mathbb{F}}$.

- Let $\lambda$ and $\lambda^{\prime}$ be two elements of $\mathbb{F}$ such that the polynomials $X^{2}+\lambda X+1 \in \mathbb{F}[X]$ and $X^{2}+\lambda^{\prime} X+1 \in \mathbb{F}[X]$ are irreducible. Then the two trivectors $\chi_{B 5}(\lambda)$ and $\chi_{B 5}\left(\lambda^{\prime}\right)$ are quasi-Sp $(V, f)$-equivalent if and only if the polynomials $X^{2}+\lambda X+1$ and $X^{2}+\lambda^{\prime} X+1$ define the same quadratic extension of $\mathbb{F}$ in $\overline{\mathbb{F}}$.

- Let $\lambda, \lambda^{\prime} \in \mathbb{F}^{*}$. Then the two trivectors $\chi_{C 1}(\lambda)$ and $\chi_{C 1}\left(\lambda^{\prime}\right)$ are quasi-Sp $(V, f)$ equivalent if and only if $\lambda=\lambda^{\prime}$.

- Let $\lambda, \lambda^{\prime} \in \mathbb{F}^{*}$. Then the two trivectors $\chi_{D 2}(\lambda)$ and $\chi_{D 2}\left(\lambda^{\prime}\right)$ are quasi-Sp $(V, f)$ equivalent if and only if $\lambda=\lambda^{\prime}$.

- Let $\lambda_{1}, \lambda_{2}, \lambda_{1}^{\prime}, \lambda_{2}^{\prime} \in \mathbb{F}^{*}$ such that none of the equations $\lambda_{1} X^{2}+\lambda_{2} Y^{2}+Z^{2}=0$ and $\lambda_{1}^{\prime} X^{2}+\lambda_{2}^{\prime} Y^{2}+Z^{2}=0$ has solutions for $(X, Y, Z) \in \mathbb{F}^{3} \backslash\{(0,0,0)\}$. Then the two trivectors $\chi_{D 3}\left(\lambda_{1}, \lambda_{2}\right)$ and $\chi_{D 3}\left(\lambda_{1}^{\prime}, \lambda_{2}^{\prime}\right)$ are quasi-Sp $(V, f)$-equivalent if and only if there exists a $\mu \in \mathbb{F}^{*}$ such that the matrices $\operatorname{diag}\left(\mu \lambda_{1}, \mu \lambda_{2}, \mu\right)$ and $\operatorname{diag}\left(\lambda_{1}^{\prime}, \lambda_{2}^{\prime}, 1\right)$ are pseudo-congruent. 
- Let $\lambda_{1}, \lambda_{2}, \lambda_{1}^{\prime}, \lambda_{2}^{\prime} \in \mathbb{F}^{*}$. Then the two trivectors $\chi_{D 4}\left(\lambda_{1}, \lambda_{2}\right)$ and $\chi_{D 4}\left(\lambda_{1}^{\prime}, \lambda_{2}^{\prime}\right)$ are quasi-Sp $(V, f)$-equivalent if and only if there exists $a \mu \in \mathbb{F}^{*}$ such that the matrices

$$
\left[\begin{array}{ccc}
\mu \lambda_{1} & 0 & \mu \lambda_{1} \\
0 & \mu \lambda_{2} & 0 \\
0 & 0 & \mu
\end{array}\right] \quad \text { and } \quad\left[\begin{array}{ccc}
\lambda_{1}^{\prime} & 0 & \lambda_{1}^{\prime} \\
0 & \lambda_{2}^{\prime} & 0 \\
0 & 0 & 1
\end{array}\right]
$$

are pseudo-congruent.

It should be mentioned that the classification of the quasi-Sp $(V, f)$-equivalence classes is incomplete in the characteristic 2 case, as it does not include the case of trivectors of Type (E). It could be that (for certain fields) some (if not all) of the trivectors of Type (E) are quasi- $S p(V, f)$-equivalent with a trivector of Type $(\mathrm{A}),(\mathrm{B}),(\mathrm{C})$ or $(\mathrm{D})$. In the final remark of this paper, we will show that this is always the case for some of the trivectors of Type (E).

Suppose $\mathbb{F}$ is a finite field of order $q$. For $q$ odd, a classification of all quasi- $S p(V, f)$ equivalence classes (or equivalently, $S p(V, f)$-equivalence classes contained in $W$ ) can be found in [9] along with information about the stabilizers of the trivectors. For $q$ even, $\mathbb{F}$ is a perfect field. For perfect fields of characteristic 2, it can be shown that any nonzero trivector is quasi- $S p(V, f)$-equivalent with a trivector of Type (A), (B) or (C) (see [10] for a discussion using the connection with hyperplanes of $D W(5, \mathbb{F})$ ) and so our results here along with [10] offer a complete classification of the quasi- $S p(V, f)$-equivalence classes for those fields.

The classification results obtained in the present paper rely on the classification of the $S p(V, f)$-equivalence classes of trivectors of $V$. This lengthy classification, which was realized in a series of four papers $[13,14,15,16]$ by the authors, will be recalled in Section 2. This classification will immediately be used in Section 2 to determine all quasi- $S p(V, f)$-equivalence classes in the case $\operatorname{char}(\mathbb{F}) \neq 2$. The case $\operatorname{char}(\mathbb{F})=2$ is more complicated and will be treated in Section 4. Section 3 is devoted to developing the tools that will be necessary to obtain the classification results in the characteristic 2 case.

\section{The classification of the $S p(V, f)$-equivalence classes of trivectors}

We continue with the notation introduced in Section 1. So, $V$ denotes a 6-dimensional vector space over a field $\mathbb{F}$ equipped with a nondegenerate alternating bilinear form $f$, and $\left(e_{1}, f_{1}, e_{2}, f_{2}, e_{3}, f_{3}\right)$ denotes a fixed hyperbolic basis of $(V, f)$. In a series of four papers $([13,14,15,16])$, the authors obtained a complete classification of all $S p(V, f)$-equivalence classes of nonzero trivectors of $V$. This classification is summarized in Tables 1 and 2 .

A trivector of $V$ is said to be of Type $(X) \in\left\{(A 1),(A 2), \ldots,\left(E 2^{\prime}\right),\left(E 3^{\prime}\right)\right\}$ if it is $S p(V, f)$ equivalent with (one of) the trivector(s) mentioned in $(\mathrm{X})$ of the tables. The trivectors 


\begin{tabular}{|c|c|}
\hline$(\mathrm{A} 1)$ & $\chi_{A 1}:=e_{1} \wedge e_{2} \wedge e_{3}$ \\
\hline$(\mathrm{A} 2)$ & $\chi_{A 2}:=e_{1} \wedge e_{2} \wedge f_{2}$ \\
\hline (B1) & $\chi_{B 1}:=e_{1} \wedge e_{2} \wedge e_{3}+e_{1} \wedge f_{1} \wedge f_{3}$ \\
\hline$(\mathrm{B} 2)$ & $\chi_{B 2}:=e_{1} \wedge e_{2} \wedge f_{2}+e_{1} \wedge f_{1} \wedge e_{3}$ \\
\hline$(\mathrm{B} 3)$ & $\chi_{B 3}:=e_{1} \wedge e_{2} \wedge f_{2}+e_{1} \wedge e_{3} \wedge f_{3}$ \\
\hline$(\mathrm{B} 4)$ & $\begin{array}{l}\chi_{B 4}(\lambda):=e_{1} \wedge e_{2} \wedge e_{3}+\lambda \cdot e_{1} \wedge f_{2} \wedge f_{3} \text { for some } \lambda \in \mathbb{F}^{*} \\
\lambda / \lambda^{\prime} \text { is a square in } \mathbb{F}\end{array}$ \\
\hline$(\mathrm{B} 5)$ & $\begin{array}{l}\chi_{B 5}(\lambda):=\lambda \cdot e_{1} \wedge e_{2} \wedge f_{2}+e_{1} \wedge\left(e_{2}-e_{3}\right) \wedge\left(f_{2}+f_{3}\right) \text { for some } \lambda \in \mathbb{F}^{*} \\
\lambda=\lambda^{\prime}\end{array}$ \\
\hline (C1) & $\begin{array}{l}\chi_{C 1}(\lambda):=e_{1} \wedge e_{2} \wedge e_{3}+\lambda \cdot f_{1} \wedge f_{2} \wedge f_{3} \text { for some } \lambda \in \mathbb{F}^{*} \\
\lambda^{\prime} \in\{\lambda,-\lambda\}\end{array}$ \\
\hline$(\mathrm{C} 2)$ & $\begin{array}{l}\chi_{C 2}(\lambda):=f_{1} \wedge\left(e_{2}+e_{3}\right) \wedge\left(f_{2}-f_{3}\right)+\lambda \cdot e_{1} \wedge e_{2} \wedge f_{2} \text { for some } \lambda \in \mathbb{F}^{*} \\
\lambda=\lambda^{\prime}\end{array}$ \\
\hline$(\mathrm{C} 3)$ & $\begin{array}{l}\chi_{C 3}(\lambda):=e_{1} \wedge e_{2} \wedge f_{2}+\lambda \cdot f_{1} \wedge e_{3} \wedge f_{3} \text { for some } \lambda \in \mathbb{F}^{*} \\
\lambda^{\prime} \in\{\lambda,-\lambda\}\end{array}$ \\
\hline$(\mathrm{C} 4)$ & $\begin{array}{l}\chi_{C 4}(\lambda):=f_{1} \wedge e_{3} \wedge\left(e_{2}+f_{3}\right)+\lambda \cdot e_{1} \wedge e_{2} \wedge f_{2} \text { for some } \lambda \in \mathbb{F}^{*} \\
\lambda^{\prime} \in\{\lambda,-\lambda\}\end{array}$ \\
\hline (C5) & $\begin{array}{l}\chi_{C 5}(\lambda):=e_{1} \wedge e_{3} \wedge\left(f_{3}+f_{2}\right)+\lambda \cdot e_{2} \wedge f_{3} \wedge\left(f_{1}+e_{3}\right) \text { for some } \lambda \in \mathbb{F}^{*} \\
\lambda^{\prime} \in\{\lambda,-\lambda\}\end{array}$ \\
\hline$(\mathrm{C} 6)$ & $\begin{array}{l}\chi_{C 6}(\lambda, \epsilon):=f_{1} \wedge\left(e_{2}+e_{3}\right) \wedge\left(f_{2}+\epsilon f_{3}\right)+\lambda \cdot e_{1} \wedge e_{2} \wedge f_{2} \text { for some } \lambda \in \mathbb{F}^{*} \\
\text { and some } \epsilon \in \mathbb{F} \backslash\{0,-1\} \\
\epsilon=\epsilon^{\prime} \text { and } \lambda^{\prime} \in\{\lambda,-\lambda\}\end{array}$ \\
\hline (D1) & $\chi_{D 1}:=e_{1} \wedge e_{2} \wedge f_{2}+e_{2} \wedge f_{1} \wedge e_{3}+f_{1} \wedge e_{1} \wedge f_{3}$ \\
\hline$(\mathrm{D} 2)$ & $\begin{array}{l}\chi_{D 2}(\lambda):=\lambda \cdot e_{1} \wedge e_{2} \wedge f_{3}+e_{2} \wedge f_{1} \wedge e_{3}+f_{1} \wedge e_{1} \wedge f_{2} \text { for some } \lambda \in \mathbb{F}^{*} \\
\lambda=\lambda^{\prime}\end{array}$ \\
\hline (D3) & $\begin{array}{l}\chi_{D 3}\left(\lambda_{1}, \lambda_{2}\right):=e_{1} \wedge e_{2} \wedge f_{3}+\lambda_{1} \cdot e_{2} \wedge e_{3} \wedge f_{1}+\lambda_{2} \cdot e_{3} \wedge e_{1} \wedge f_{2} \text { for some } \lambda_{1}, \lambda_{2} \in \mathbb{F}^{*} \\
\text { the matrices } \operatorname{diag}\left(\lambda_{1}, \lambda_{2}, \lambda_{1} \lambda_{2}\right) \text { and } \operatorname{diag}\left(\lambda_{1}^{\prime}, \lambda_{2}^{\prime}, \lambda_{1}^{\prime} \lambda_{2}^{\prime}\right) \text { are congruent }\end{array}$ \\
\hline (D4) & $\begin{array}{l}\chi_{D 4}\left(\lambda_{1}, \lambda_{2}\right):=e_{1} \wedge e_{2} \wedge f_{3}+\lambda_{1} \cdot e_{2} \wedge e_{3} \wedge\left(f_{1}+f_{3}\right)+\lambda_{2} \cdot e_{3} \wedge e_{1} \wedge f_{2} \\
\text { for some } \lambda_{1}, \lambda_{2} \in \mathbb{F}^{*} \\
\lambda_{1}=\lambda_{1}^{\prime} \text { and there exist } X, Y \in \mathbb{F} \text { such that } X^{2}+\lambda_{1} X Y+\lambda_{1} Y^{2}=\lambda_{2}^{\prime} / \lambda_{2}\end{array}$ \\
\hline$(\bar{D} 5)$ & $\begin{array}{l}\chi_{D 5}(\lambda):=e_{1} \wedge e_{2} \wedge f_{3}+\lambda \cdot e_{2} \wedge e_{3} \wedge\left(f_{1}+f_{2}+f_{3}\right)-e_{3} \wedge e_{1} \wedge f_{2} \text { for some } \lambda \in \mathbb{F}^{*} \\
1 / \lambda+1 / \lambda^{\prime} \text { is of the form } X^{2}+X \text { for some } X \in \mathbb{F}\end{array}$ \\
\hline (D6) & (only if $\operatorname{char}(\mathbb{F}) \neq 2) \chi_{D 6}:=-e_{1} \wedge e_{2} \wedge f_{2}+e_{2} \wedge e_{3} \wedge f_{1}+e_{3} \wedge e_{1} \wedge f_{3}$ \\
\hline$(\mathrm{D} 7)$ & (only if $|\mathbb{F}|=2) \chi_{D 7}:=e_{1} \wedge e_{2} \wedge f_{2}+e_{2} \wedge e_{3} \wedge\left(f_{1}+f_{3}\right)+e_{3} \wedge e_{1} \wedge f_{3}$ \\
\hline
\end{tabular}

Table 1: The $S p(V, f)$-equivalence classes of trivectors of Type A, B, C and D 


\begin{tabular}{|c|c|}
\hline \multirow[t]{2}{*}{ (E1) } & $\begin{array}{l}\chi_{E 1}\left(a, b, h_{1}, h_{2}, h_{3}\right):=2 \cdot e_{1} \wedge e_{2} \wedge e_{3}+h_{1} h_{2} h_{3} a\left(a^{2}+3 b\right) \cdot f_{1} \wedge f_{2} \wedge f_{3}+\left(a^{2}+2 b\right) \cdot \\
\left(h_{1} h_{2} \cdot f_{1} \wedge f_{2} \wedge e_{3}+h_{1} h_{3} \cdot f_{1} \wedge e_{2} \wedge f_{3}+h_{2} h_{3} \cdot e_{1} \wedge f_{2} \wedge f_{3}\right)+a \cdot\left(h_{1} \cdot f_{1} \wedge e_{2} \wedge e_{3}\right. \\
\left.+h_{2} \cdot e_{1} \wedge f_{2} \wedge e_{3}+h_{3} \cdot e_{1} \wedge e_{2} \wedge f_{3}\right) \text { for some }(a, b) \in \Psi \text { and some } h_{1}, h_{2}, h_{3} \in \mathbb{F}^{*}\end{array}$ \\
\hline & $\begin{array}{l}(a, b)=\left(a^{\prime}, b^{\prime}\right) \text { and there exists a } 3 \times 3 \text {-matrix } A \text { over } \mathbb{F}^{\prime} \text { with } \operatorname{det}(A)=1 \text { and an } \\
\epsilon \in\{1,-1\} \text { such that } A \cdot \operatorname{diag}\left(h_{1}, h_{2}, h_{3}\right) \cdot\left(A^{\psi}\right)^{T}=\epsilon \cdot \operatorname{diag}\left(h_{1}^{\prime}, h_{2}^{\prime}, h_{3}^{\prime}\right) \text {. Here, } \mathbb{F}^{\prime} \subseteq \overline{\mathbb{F}} \\
\text { is the extension of } \mathbb{F} \text { determined by } X^{2}-a X-b \in \mathbb{F}[X] \text { and } 1 \neq \psi \in \operatorname{Gal}\left(\mathbb{F}^{\prime} / \mathbb{F}\right)\end{array}$ \\
\hline (E2) & $\begin{array}{l}\chi_{E 2}(a, b, k):=k \cdot\left(f_{1} \wedge e_{2} \wedge f_{3}-b \cdot f_{1} \wedge f_{2} \wedge e_{3}+a \cdot f_{1} \wedge e_{3} \wedge f_{3}\right) \\
+e_{1} \wedge e_{2} \wedge f_{2}+e_{1} \wedge e_{3} \wedge f_{3} \text { for some }(a, b) \in \Psi \text { and some } k \in \mathbb{F}^{*} \\
(a, b)=\left(a^{\prime}, b^{\prime}\right) \text { and } k^{\prime} \in\{k,-k\}\end{array}$ \\
\hline (E3) & $\begin{array}{l}\chi_{E 3}(a, b, k, h):=k \cdot\left(f_{1} \wedge e_{2} \wedge f_{3}-b \cdot f_{1} \wedge f_{2} \wedge e_{3}+a \cdot f_{1} \wedge e_{3} \wedge f_{3}\right)+e_{1} \wedge e_{2} \wedge f_{2} \\
+e_{1} \wedge e_{3} \wedge f_{3}+h \cdot e_{1} \wedge f_{2} \wedge f_{3} \text { for some }(a, b) \in \Psi \text { and some } k, h \in \mathbb{F}^{*} \\
(a, b)=\left(a^{\prime}, b^{\prime}\right) \text { and there exist a } \sigma \in\{1,-1\} \text { and } X, Y \in \mathbb{F} \text { such that } k^{\prime}=\sigma k \text { and } \\
h^{\prime}=\sigma h\left(X^{2}+a X Y-b Y^{2}\right)\end{array}$ \\
\hline 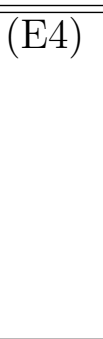 & $\begin{array}{l}\chi_{E 4}\left(a, b, k, h_{1}, h_{2}\right):=\left(1-h_{1} h_{2}\left(a^{2}+4 b\right)\right) \cdot e_{1} \wedge e_{2} \wedge f_{2}+\left(1+h_{1} h_{2}\left(a^{2}+4 b\right)\right) \cdot \\
e_{1} \wedge e_{3} \wedge f_{3}+h_{1}\left(1-h_{1} h_{2}\left(a^{2}+4 b\right)\right) \cdot e_{1} \wedge f_{2} \wedge f_{3}+\left(a^{2}+4 b\right) h_{2} \cdot e_{1} \wedge e_{2} \wedge e_{3} \\
+k \cdot\left(f_{1} \wedge e_{2} \wedge f_{3}-b\left(1-h_{1} h_{2}\left(a^{2}+4 b\right)\right) \cdot f_{1} \wedge f_{2} \wedge e_{3}+a \cdot f_{1} \wedge e_{3} \wedge f_{3}\right) \\
\text { for some }(a, b) \in \Psi \text { and some } k, h_{1}, h_{2} \in \mathbb{F}^{*} \text { satisfying } h_{1} h_{2}\left(a^{2}+4 b\right) \neq 1 \\
(a, b)=\left(a^{\prime}, b^{\prime}\right), h_{1} h_{2}=h_{1}^{\prime} h_{2}^{\prime} \text { and there exist } X, Y, Z, U \in \mathbb{F} \text { and a } \sigma \in\{1,-1\} \text { such } \\
\text { that } k^{\prime}=\sigma k \text { and } \sigma h_{1}^{\prime}=h_{1}\left(X^{2}+a X Y-b Y^{2}\right)+h_{2}\left(Z^{2}+a Z U-b U^{2}\right)\end{array}$ \\
\hline (E5) & $\begin{array}{l}\chi_{E 5}(a, b, k):=f_{1} \wedge e_{2} \wedge f_{3}+2 \cdot e_{1} \wedge f_{1} \wedge e_{2}-a \cdot f_{1} \wedge e_{2} \wedge f_{2}+a \cdot f_{1} \wedge e_{3} \wedge f_{3} \\
+\left(a^{2}+b\right) \cdot f_{1} \wedge f_{2} \wedge e_{3}+k \cdot\left(a \cdot e_{1} \wedge f_{2} \wedge e_{3}-e_{1} \wedge e_{2} \wedge f_{2}+e_{1} \wedge e_{3} \wedge f_{3}\right) \\
+a \cdot e_{1} \wedge f_{1} \wedge e_{3} \text { for some }(a, b) \in \Psi \text { and some } k \in \mathbb{F}^{*} \\
(a, b)=\left(a^{\prime}, b^{\prime}\right) \text { and } k^{\prime} \in\{k,-k\}\end{array}$ \\
\hline$\overline{\left(\mathrm{E} 1{ }^{\prime}\right)}$ & $\begin{array}{l}\chi_{E 1}^{\prime}\left(a, h_{1}, h_{2}, h_{3}\right):=\frac{a+1}{a} \cdot e_{1} \wedge e_{2} \wedge e_{3}+\left(e_{1}+h_{1} f_{1}\right) \wedge\left(e_{2}+h_{2} f_{2}\right) \wedge\left(e_{3}+h_{3} f_{3}\right) \\
+(a+1) h_{1} h_{2} h_{3} \cdot f_{1} \wedge f_{2} \wedge f_{3} \text { for some } a \in \Psi^{\prime} \text { and some } h_{1}, h_{2}, h_{3} \in \mathbb{F}^{*} \\
a=a^{\prime} \text { and there exists a } 3 \times 3 \text {-matrix } A \text { over } \mathbb{F}^{\prime} \text { with } \operatorname{det}(A)=1 \text { such that } \\
\operatorname{diag}\left(h_{1}^{\prime}, h_{2}^{\prime}, h_{3}^{\prime}\right)=A \cdot \operatorname{diag}\left(h_{1}, h_{2}, h_{3}\right) \cdot A^{T} . \text { Here, } \mathbb{F}^{\prime} \subseteq \overline{\mathbb{F}} \text { is the extension of } \mathbb{F} \\
\text { determined by } X^{2}+a \in \mathbb{F}[X]\end{array}$ \\
\hline$\overline{(\mathrm{E} 2 ')}$ & $\begin{array}{l}\chi_{E 2}^{\prime}\left(a, k, h_{1}, h_{2}\right):=\frac{1}{a} \cdot e_{1} \wedge\left(e_{2}+h_{1}(a+1) f_{3}\right) \wedge f_{2}+k \cdot f_{1} \wedge e_{3} \wedge\left(h_{2}(a+1) e_{2}+f_{3}\right) \\
+\frac{1}{(a+1)^{2}} \cdot\left(e_{1}+k f_{1}\right) \wedge\left(e_{2}+(a+1) e_{3}+h_{1}(a+1) f_{3}\right) \wedge\left(h_{2}(a+1) e_{2}+(a+1) f_{2}+f_{3}\right) \\
\text { for some } a \in \Psi^{\prime} \text { and some } k, h_{1}, h_{2} \in \mathbb{F} \text { satisfying } k \neq 0 \text { and } h_{1} h_{2}(a+1)^{2} \neq 1 \\
a=a^{\prime}, k=k^{\prime}, h_{1} h_{2}=h_{1}^{\prime} h_{2}^{\prime} \text { and there exist } X, Y, Z, U \in \mathbb{F} \text { such that } \\
h_{1}^{\prime}=h_{1}\left(X^{2}+a Y^{2}\right)+h_{2}\left(Z^{2}+a U^{2}\right)+(X U+Y Z)\end{array}$ \\
\hline$\overline{\left(\mathrm{E} 33^{\prime}\right)}$ & $\begin{array}{l}\chi_{E 3}^{\prime}\left(a, h_{1}, h_{2}\right):=\frac{1}{a} \cdot e_{1} \wedge\left(e_{2}+e_{3}\right) \wedge f_{2}+e_{2} \wedge f_{1} \wedge\left(e_{1}+h_{1} f_{3}\right)+\frac{1}{a+1} \cdot\left(e_{1}+e_{2}\right) \wedge \\
\left(e_{3}+h_{1} f_{3}\right) \wedge\left((a+1)^{2} h_{2} e_{1}+f_{1}+f_{2}\right) \text { for some } a \in \Psi^{\prime} \text { and some }\left(h_{1}, h_{2}\right) \in \mathbb{F}^{*} \times \mathbb{F}\end{array}$ \\
\hline & me $X, Y \in \mathbb{F}$ \\
\hline
\end{tabular}

Table 2: The $S p(V, f)$-equivalence classes of trivectors of Type E 
of Type (D7) only exist if $|\mathbb{F}|=2$, which seems somewhat unnatural. The reason is that we have aimed to give a classification which is valid for all characteristics. If we had only restricted to the characteristic 2 case, a "more natural" description can be given by replacing (D5) and (D7) by the following:

$\left(D 5^{\prime}\right) \chi_{D 5}(\lambda):=e_{1} \wedge e_{2} \wedge f_{3}+\lambda \cdot e_{2} \wedge e_{3} \wedge\left(f_{1}+f_{2}+f_{3}\right)-e_{3} \wedge e_{1} \wedge f_{2}$ for some $\lambda \in \mathbb{F}^{*}$ such that $\lambda^{-1}$ is not of the form $X^{2}+X$ for some $X \in \mathbb{F}$;

$$
\chi_{D 7}:=e_{1} \wedge e_{2} \wedge f_{2}+e_{2} \wedge e_{3} \wedge\left(f_{1}+f_{3}\right)+e_{3} \wedge e_{1} \wedge f_{3}
$$

The two tables divide the nonzero trivectors of $V$ into 28 families (which are subfamilies of those mentioned in Proposition 1.1; hence the names for the types). In [13, 14, 15, 16], it was also shown that trivectors belonging to distinct families can never be $S p(V, f)$ equivalent. In case a family of trivectors has at least two members, the tables also mention a condition that indicates when two trivectors of the same family are equivalent. We illustrate the interpretation of that condition by means of a concrete example, namely that of the (E3)-family: If $(a, b),\left(a^{\prime}, b^{\prime}\right) \in \Psi$ and $k, h, k^{\prime}, h^{\prime} \in \mathbb{F}^{*}$, then the two trivectors $\chi_{E 3}(a, b, k, h)$ and $\chi_{E 3}\left(a^{\prime}, b^{\prime}, k^{\prime}, h^{\prime}\right)$ are $S p(V, f)$-equivalent if and only if $(a, b)=\left(a^{\prime}, b^{\prime}\right)$ and there exist a $\sigma \in\{1,-1\}$ and $X, Y \in \mathbb{F}$ such that $k^{\prime}=\sigma k$ and $h^{\prime}=\sigma h\left(X^{2}+a X Y-b Y^{2}\right)$ (we use accents for the parameters of the second member of the family).

The subspace $W$ defined in Section 1 is the subspace of $\wedge^{3} V$ generated by the 14 trivectors $e_{1} \wedge e_{2} \wedge e_{3}, e_{1} \wedge e_{2} \wedge f_{3}, e_{1} \wedge f_{2} \wedge e_{3}, e_{1} \wedge f_{2} \wedge f_{3}, f_{1} \wedge e_{2} \wedge e_{3}, f_{1} \wedge e_{2} \wedge f_{3}, f_{1} \wedge f_{2} \wedge e_{3}$, $f_{1} \wedge f_{2} \wedge f_{3}, e_{1} \wedge\left(e_{2} \wedge f_{2}-e_{3} \wedge f_{3}\right), f_{1} \wedge\left(e_{2} \wedge f_{2}-e_{3} \wedge f_{3}\right), e_{2} \wedge\left(e_{1} \wedge f_{1}-e_{3} \wedge f_{3}\right)$, $f_{2} \wedge\left(e_{1} \wedge f_{1}-e_{3} \wedge f_{3}\right), e_{3} \wedge\left(e_{1} \wedge f_{1}-e_{2} \wedge f_{2}\right), f_{3} \wedge\left(e_{1} \wedge f_{1}-e_{2} \wedge f_{2}\right)$. So, the nonzero trivectors contained in $W$ are precisely the trivectors of Type A1, B4, C1, D3, E1, E1' and (only if the characteristic of $\mathbb{F}$ is equal to 2 ) B3.

The subspace $\widetilde{W}$ defined in Section 1 is the subspace of $\wedge^{3} V$ generated by the 6 trivectors $e_{1} \wedge\left(e_{2} \wedge f_{2}+e_{3} \wedge f_{3}\right), f_{1} \wedge\left(e_{2} \wedge f_{2}+e_{3} \wedge f_{3}\right), e_{2} \wedge\left(e_{1} \wedge f_{1}+e_{3} \wedge f_{3}\right), f_{2} \wedge\left(e_{1} \wedge f_{1}+e_{3} \wedge f_{3}\right)$, $e_{3} \wedge\left(e_{1} \wedge f_{1}+e_{2} \wedge f_{2}\right), f_{3} \wedge\left(e_{1} \wedge f_{1}+e_{2} \wedge f_{2}\right)$. The nonzero trivectors contained in $\widetilde{W}$ are precisely the trivectors of Type B3.

Suppose now that $\operatorname{char}(\mathbb{F}) \neq 2$. Then every trivector $\chi \in \bigwedge^{3} V$ can be written in a unique way as $\chi_{1}+\chi_{2}$ where $\chi_{1} \in W$ and $\chi_{2} \in \widetilde{W}$. We define $\pi_{W}(\chi):=\chi_{1}$ and $\pi_{\widetilde{W}}(\chi):=\chi_{2}$. For every $\theta \in S p(V, f)$ and every $\chi \in \bigwedge^{3} V$, we have $\theta(\chi)=\theta\left(\pi_{W}(\chi)\right)+\theta\left(\pi_{\widetilde{W}}(\chi)\right)$ where $\theta\left(\pi_{W}(\chi)\right) \in W$ and $\theta\left(\pi_{\widetilde{W}}(\chi)\right) \in \widetilde{W}$. So, if $\theta \in S p(V, f)$, then $\theta \circ \pi_{W}=\pi_{W} \circ \theta$ and $\theta \circ \pi_{\widetilde{W}}=\pi_{\widetilde{W}} \circ \theta$.

Proposition 2.1 Suppose char $(\mathbb{F}) \neq 2$. Then every trivector $\chi$ of $V$ is quasi-Sp $(V, f)$ equivalent with $\pi_{W}(\chi)$. Moreover, two trivectors $\chi_{1}$ and $\chi_{2}$ of $V$ are quasi-Sp $(V, f)$ equivalent if and only if $\pi_{W}\left(\chi_{1}\right)$ and $\pi_{W}\left(\chi_{2}\right)$ are $S p(V, f)$-equivalent.

Proof. The first claim follows from the fact that $\chi=\pi_{W}(\chi)+\pi_{\widetilde{W}}(\chi)$, where $\pi_{\widetilde{W}}(\chi) \in \widetilde{W}$. As for the second claim, the two trivectors $\pi_{W}\left(\chi_{1}\right)$ and $\pi_{W}\left(\chi_{2}\right)$ are $S p(V, f)$-equivalent if 
and only if $\pi_{W}\left(\chi_{2}\right)=\theta\left(\pi_{W}\left(\chi_{1}\right)\right)=\pi_{W}\left(\theta\left(\chi_{1}\right)\right)$ for some $\theta \in S p(V, f)$, i.e. if and only if there exist a $\theta \in S p(V, f)$ and a $\chi \in \widetilde{W}$ such that $\chi_{2}=\theta\left(\chi_{1}\right)+\chi$.

Theorems 1.2 and 1.3 are immediate consequences of Proposition 2.1 and the classification mentioned in Tables 1 and 2 .

\section{Tools}

We continue with the notation introduced in Section 1. Recall that $V$ is a 6-dimensional vector space over a field $\mathbb{F}$ equipped with a nondegenerate alternating bilinear form $f$.

The following lemma will be useful during the classification of the quasi- $S p(V, f)$ equivalence classes of trivectors in the case $\operatorname{char}(\mathbb{F})=2$. A proof of it can be found in De Bruyn and Kwiatkowski [13, Lemma 2.9].

Lemma 3.1 ([13]) Let $U$ be a 4-dimensional vector space over the field $\mathbb{F}$ and let $\left\{u_{1}, u_{2}\right.$, $\left.u_{3}, u_{4}\right\},\left\{u_{1}^{\prime}, u_{2}^{\prime}, u_{3}^{\prime}, u_{4}^{\prime}\right\}$ be two bases of $U$ such that $u_{1} \wedge u_{2}+u_{3} \wedge u_{4}=u_{1}^{\prime} \wedge u_{2}^{\prime}+u_{3}^{\prime} \wedge u_{4}^{\prime}$. Then $u_{1} \wedge u_{2} \wedge u_{3} \wedge u_{4}=u_{1}^{\prime} \wedge u_{2}^{\prime} \wedge u_{3}^{\prime} \wedge u_{4}^{\prime}$.

If $\left(e_{1}, f_{1}, e_{2}, f_{2}, e_{3}, f_{3}\right)$ is a hyperbolic basis of $(V, f)$, then

(1) for every permutation $\sigma$ of $\{1,2,3\}$, also $\left(e_{\sigma(1)}, f_{\sigma(1)}, e_{\sigma(2)}, f_{\sigma(2)}, e_{\sigma(3)}, f_{\sigma(3)}\right)$ is a hyperbolic basis of $(V, f)$;

(2) for every $\lambda \in \mathbb{F}^{*}$, also $\left(\frac{e_{1}}{\lambda}, \lambda f_{1}, e_{2}, f_{2}, e_{3}, f_{3}\right)$ is a hyperbolic basis of $(V, f)$;

(3) for every $\lambda \in \mathbb{F}$, also $\left(e_{1}+\lambda e_{2}, f_{1}, e_{2},-\lambda f_{1}+f_{2}, e_{3}, f_{3}\right)$ is a hyperbolic basis of $(V, f)$;

(4) for every $\lambda \in \mathbb{F}$, also $\left(e_{1}, f_{1}, e_{2}, f_{2}, e_{3}, f_{3}+\lambda e_{3}\right)$ is a hyperbolic basis of $(V, f)$;

(5) for every $\lambda \in \mathbb{F}$, also $\left(e_{1}, f_{1}, e_{2}, f_{2}, e_{3}+\lambda f_{3}, f_{3}\right)$ is a hyperbolic basis of $(V, f)$.

For every $i \in\{1,2, \ldots, 5\}$, let $\Omega_{i}$ denote the set of all ordered pairs $\left(B_{1}, B_{2}\right)$ of hyperbolic bases of $(V, f)$ such that $B_{2}$ can be obtained from $B_{1}$ as described in $(i)$ above. The following lemma was proved in De Bruyn [11, Lemma 2.1].

Lemma 3.2 ([11]) If $B$ and $B^{\prime}$ are two hyperbolic bases of $(V, f)$, then there exist hyperbolic bases $B_{0}, B_{1}, \ldots, B_{k}$ of $(V, f)$ for some $k \geq 0$ such that $B_{0}=B, B_{k}=B^{\prime}$ and $\left(B_{i-1}, B_{i}\right) \in \Omega_{1} \cup \Omega_{2} \cup \cdots \cup \Omega_{5}$ for every $i \in\{1,2, \ldots, k\}$.

The properties mentioned in Lemmas 3.3 and 3.4 below are known. (One could give explicit descriptions of $\pi$ and $\pi^{\prime}$ with respect to those bases of $\bigwedge^{3} V$ and $\bigwedge^{4} V$ defined by some specific hyperbolic basis $\left(e_{1}, f_{1}, e_{2}, f_{2}, e_{3}, f_{3}\right)$ of $(V, f)$ and use Lemma 3.2 to show that these descriptions are independent of the chosen hyperbolic basis.) 
Lemma 3.3 There exists a unique linear map $\pi: \bigwedge^{3} V \rightarrow V$ for which $\pi(W)=\{o\}$ and $\pi\left(e_{1} \wedge e_{2} \wedge f_{2}\right)=e_{1}$ for any three linearly independent vectors $e_{1}, e_{2}, f_{2} \in V$ satisfying $f\left(e_{1}, e_{2}\right)=f\left(e_{1}, f_{2}\right)=0$ and $f\left(e_{2}, f_{2}\right)=1$.

Lemma 3.4 There exists a unique linear map $\pi^{\prime}: \bigwedge^{4} V \rightarrow \bigwedge^{2} V$ mapping

- $e_{1} \wedge f_{1} \wedge e_{2} \wedge e_{3}$ to $e_{2} \wedge e_{3}$ for any four linearly independent vectors $e_{1}, f_{1}, e_{2}$ and $e_{3}$ of $V$ satisfying $f\left(e_{1}, f_{1}\right)=1, f\left(e_{2}, e_{3}\right)=0$ and $\left\{e_{1}, f_{1}\right\} \subseteq e_{2}^{\perp_{f}} \cap e_{3}^{\perp_{f}}$;

- $e_{1} \wedge f_{1} \wedge e_{2} \wedge f_{2}$ to $e_{1} \wedge f_{1}+e_{2} \wedge f_{2}$ for any four linearly independent vectors $e_{1}, f_{1}$, $e_{2}$ and $f_{2}$ of $V$ satisfying $f\left(e_{1}, f_{1}\right)=f\left(e_{2}, f_{2}\right)=1$ and $\left\{e_{1}, f_{1}\right\} \subseteq e_{2}^{\perp_{f}} \cap f_{2}^{\perp_{f}}$.

Above, we have already mentioned that $V$ and $\widetilde{W}$ are isomorphic as $S p(V, f)$-modules. We now describe an explicit isomorphism. If $v$ is a nonzero vector of $V$, then we define

$$
\phi(v):=v \wedge\left(e_{2} \wedge f_{2}+e_{3} \wedge f_{3}\right),
$$

where the vectors $e_{2}, f_{2}, e_{3}, f_{3}$ are chosen in such a way that $\left(v, w, e_{2}, f_{2}, e_{3}, f_{3}\right)$ is a hyperbolic basis of $(V, f)$ for a certain vector $w \in V$. It can be shown that $\phi(v)$ is independent of the chosen hyperbolic basis $\left(v, w, e_{2}, f_{2}, e_{3}, f_{3}\right)$ of $(V, f)$. We also put $\phi(o)$ equal to the zero vector of $\bigwedge^{3} V$. Then $\phi: V \rightarrow \widetilde{W}$ is a linear isomorphism between the 6-dimensional vector spaces $V$ and $\widetilde{W}$, and $\phi \circ \theta=\theta \circ \phi$ for every $\theta \in S p(V, f)$.

The following lemma is a combination of Lemma 5.4 and Corollary 5.5 of De Bruyn and Kwiatkowski [15].

Lemma 3.5 ([15]) Let $A$ and $A^{\prime}$ be two nonsingular $(3 \times 3)$-matrices over $\mathbb{F}$, and let $\left(e_{1}, f_{1}, e_{2}, f_{2}, e_{3}, f_{3}\right)$ be a hyperbolic basis of $(V, f)$. Put $\left[w_{1}, w_{2}, w_{3}\right]^{T}:=A \cdot\left[f_{1}, f_{2}, f_{3}\right]^{T}$ and $\left[w_{1}^{\prime}, w_{2}^{\prime}, w_{3}^{\prime}\right]^{T}:=A^{\prime} \cdot\left[f_{1}, f_{2}, f_{3}\right]^{T}$. Then $e_{1} \wedge e_{2} \wedge w_{3}+e_{2} \wedge e_{3} \wedge w_{1}+e_{3} \wedge e_{1} \wedge w_{2}$ and $e_{1} \wedge e_{2} \wedge w_{3}^{\prime}+e_{2} \wedge e_{3} \wedge w_{1}^{\prime}+e_{3} \wedge e_{1} \wedge w_{2}^{\prime}$ are $S p(V, f)$-equivalent if and only if one of the following two equivalent properties are satisfied:

- there exists a nonsingular $(3 \times 3)$-matrix $M$ over $\mathbb{F}$ such that $A^{\prime}=\frac{1}{\operatorname{det}(M)} \cdot M A M^{T}$;

- the matrices $\frac{A}{\operatorname{det}(A)}$ and $\frac{A^{\prime}}{\operatorname{det}\left(A^{\prime}\right)}$ are congruent.

For every $v \in V \backslash\{o\}$, let $W_{v}$ denote the subspace of $\Lambda^{3} V$ generated by all trivectors $v_{1} \wedge v_{2} \wedge v_{3}$, where $v_{1}, v_{2}, v_{3} \in V$ such that $\left\langle v_{1}, v_{2}, v_{3}\right\rangle$ is a totally isotropic subspace containing $v$.

Lemma 3.6 For every $v \in V \backslash\{o\}$, we have $\operatorname{dim}\left(W_{v}\right)=5$.

Proof. This is a known fact. If we choose a hyperbolic basis $\left(e_{1}, f_{1}, e_{2}, f_{2}, e_{3}, f_{3}\right)$ of $(V, f)$ such that $v=e_{1}$, then we would have $W_{v}=\left\langle e_{1} \wedge e_{2} \wedge e_{3}, e_{1} \wedge e_{2} \wedge f_{3}, e_{1} \wedge f_{2} \wedge e_{3}, e_{1} \wedge f_{2} \wedge\right.$ $\left.f_{3}, e_{1} \wedge\left(e_{2} \wedge f_{2}-e_{3} \wedge f_{3}\right)\right\rangle$. 
Lemma 3.7 Let $v \in V \backslash\{o\}$ and $\left\langle v_{1}^{(1)}, v_{2}^{(1)}, v_{3}^{(1)}\right\rangle$ a totally isotropic 3-dimensional subspace containing $v$. Then there exist three 3-dimensional totally isotropic subspaces $\left\langle v_{1}^{(2)}, v_{2}^{(2)}\right.$, $\left.v_{3}^{(2)}\right\rangle,\left\langle v_{1}^{(3)}, v_{2}^{(3)}, v_{3}^{(3)}\right\rangle$ and $\left\langle v_{1}^{(4)}, v_{2}^{(4)}, v_{3}^{(4)}\right\rangle$ containing $v$ and intersecting $\left\langle v_{1}^{(1)}, v_{2}^{(1)}, v_{3}^{(1)}\right\rangle$ in $a$ subspace of dimension 2 such that $\operatorname{dim}\left(\left\langle v_{1}^{(j)} \wedge v_{2}^{(j)} \wedge v_{3}^{(j)} \mid j \in\{1,2,3,4\}\right\rangle\right)=4$. Moreover, for any three totally isotropic subspaces that have been chosen in this way the following holds: if $\left\langle v_{1}^{(5)}, v_{2}^{(5)}, v_{3}^{(5)}\right\rangle$ is a 3-dimensional totally isotropic subspace intersecting $\left\langle v_{1}^{(1)}, v_{2}^{(1)}, v_{3}^{(1)}\right\rangle$ in $\langle v\rangle$, then $W_{v}=\left\langle v_{1}^{(j)} \wedge v_{2}^{(j)} \wedge v_{3}^{(j)} \mid j \in\{1,2,3,4,5\}\right\rangle$.

Proof. If the claim of the lemma is valid if $\left(v, v_{1}^{(1)}, v_{2}^{(1)}, v_{3}^{(1)}\right)=\left(w, w_{1}, w_{2}, w_{3}\right)$, then the claim is also valid if $\left(v, v_{1}^{(1)}, v_{2}^{(1)}, v_{3}^{(1)}\right)$ were equal to $\left(w, w_{1}^{\prime}, w_{2}^{\prime}, w_{3}^{\prime}\right)$, where $w_{1}^{\prime}, w_{2}^{\prime}$ and $w_{3}^{\prime}$ are three vectors of $V$ such that $\left\langle w_{1}^{\prime}, w_{2}^{\prime}, w_{3}^{\prime}\right\rangle=\left\langle w_{1}, w_{2}, w_{3}\right\rangle$. So, without loss of generality, we may suppose that $v=v_{1}^{(1)}$. Now, we can choose a hyperbolic basis $\left(e_{1}, f_{1}, e_{2}, f_{2}, e_{3}, f_{3}\right)$ of $(V, f)$ such that $v_{1}^{(1)}=e_{1}, v_{2}^{(1)}=e_{2}$ and $v_{3}^{(1)}=e_{3}$. Then $W_{v}=$ $\left\langle e_{1} \wedge e_{2} \wedge e_{3}, e_{1} \wedge e_{2} \wedge f_{3}, e_{1} \wedge f_{2} \wedge e_{3}, e_{1} \wedge f_{2} \wedge f_{3}, e_{1} \wedge\left(e_{2} \wedge f_{2}-e_{3} \wedge f_{3}\right)\right\rangle$. If $\left\langle v_{1}^{(2)}, v_{2}^{(2)}\right.$, $\left.v_{3}^{(2)}\right\rangle,\left\langle v_{1}^{(3)}, v_{2}^{(3)}, v_{3}^{(3)}\right\rangle$ and $\left\langle v_{1}^{(4)}, v_{2}^{(4)}, v_{3}^{(4)}\right\rangle$ are three 3-dimensional totally isotropic subspaces containing $v$ and intersecting $\left\langle v_{1}^{(1)}, v_{2}^{(1)}, v_{3}^{(1)}\right\rangle$ in subspaces of dimension 2 , then we have $\left\langle v_{1}^{(j)} \wedge v_{2}^{(j)} \wedge v_{3}^{(j)} \mid j \in\{1,2,3,4\}\right\rangle \subseteq\left\langle e_{1} \wedge e_{2} \wedge e_{3}, e_{1} \wedge e_{2} \wedge f_{3}, e_{1} \wedge f_{2} \wedge e_{3}, e_{1} \wedge\left(e_{2} \wedge f_{2}-e_{3} \wedge f_{3}\right)\right\rangle$. So, we must show that we can choose these subspaces in such a way that we have equality. This is realized by making the following choices:

$v_{1}^{(2)}=v_{1}^{(3)}=v_{1}^{(4)}=e_{1}, v_{2}^{(2)}=e_{2}, v_{3}^{(2)}=f_{3}, v_{2}^{(3)}=f_{2}, v_{3}^{(3)}=e_{3}, v_{2}^{(4)}=e_{2}+e_{3}, v_{3}^{(4)}=f_{2}-f_{3}$.

If $\left\langle v_{1}^{(5)}, v_{2}^{(5)}, v_{3}^{(5)}\right\rangle$ is a 3-dimensional totally isotropic subspace intersecting $\left\langle v_{1}^{(1)}, v_{2}^{(1)}, v_{3}^{(1)}\right\rangle$ in $\langle v\rangle$, then $v_{1}^{(5)} \wedge v_{2}^{(5)} \wedge v_{3}^{(5)}$, written as a linear combination of the trivectors $e_{1} \wedge e_{2} \wedge e_{3}$, $e_{1} \wedge e_{2} \wedge f_{3}, e_{1} \wedge f_{2} \wedge e_{3}, e_{1} \wedge f_{2} \wedge f_{3}$ and $e_{1} \wedge\left(e_{2} \wedge f_{2}-e_{3} \wedge f_{3}\right)$, should have a nonzero component in $e_{1} \wedge f_{2} \wedge f_{3}$, implying that $W_{v}=\left\langle v_{1}^{(j)} \wedge v_{2}^{(j)} \wedge v_{3}^{(j)} \mid j \in\{1,2,3,4,5\}\right\rangle$.

For every $\chi \in \Lambda^{3} V$, let $\mathcal{D}(\chi)$ denote the set of all $v \in V$ such that $\chi \wedge \chi^{\prime}=0$ for every $\chi^{\prime} \in W_{v}$, i.e. the set of all $v \in V$ such that $\chi \wedge v_{1} \wedge v_{2} \wedge v_{3}=0$ for all $v_{1}, v_{2}, v_{3} \in V$ such that $\left\langle v_{1}, v_{2}, v_{3}\right\rangle$ is a totally isotropic subspace containing $v$. Notice that $v \in \mathcal{D}(\chi)$ for every $v \in V$ such that $\chi \wedge v=0$. However, it is also possible that $v \in \mathcal{D}(\chi)$ while $\chi \wedge v \neq 0$.

Lemma 3.8 For every $\chi \in \bigwedge^{3} V$ and every $\chi^{\prime} \in \widetilde{W}$, we have $\mathcal{D}(\chi)=\mathcal{D}\left(\chi+\chi^{\prime}\right)$.

Proof. This follows from the fact that $\chi^{\prime} \wedge v_{1} \wedge v_{2} \wedge v_{3}=0$ for all vectors $v_{1}, v_{2}, v_{3}$ of $V$ such that $\left\langle v_{1}, v_{2}, v_{3}\right\rangle$ is totally isotropic.

Lemma 3.9 If $\chi_{1}$ and $\chi_{2}$ are two quasi-Sp $(V, f)$-equivalent trivectors of $V$, then there exists a $\theta \in S p(V, f)$ such that $\mathcal{D}\left(\chi_{2}\right)=\theta\left(\mathcal{D}\left(\chi_{1}\right)\right)$.

Proof. Suppose $\chi_{1}$ and $\chi_{2}$ are two quasi- $S p(V, f)$-equivalent trivectors. Then there exists a $\theta \in S p(V, f)$ and a $\chi \in \widetilde{W}$ such that $\chi_{2}=\theta\left(\chi_{1}\right)+\chi$. Observe that $\chi_{1} \wedge v_{1} \wedge v_{2} \wedge v_{3}=$ 
$0 \Leftrightarrow \theta\left(\chi_{1}\right) \wedge \theta\left(v_{1}\right) \wedge \theta\left(v_{2}\right) \wedge \theta\left(v_{3}\right)=0, v \in\left\langle v_{1}, v_{2}, v_{3}\right\rangle \Leftrightarrow \theta(v) \in\left\langle\theta\left(v_{1}\right), \theta\left(v_{2}\right), \theta\left(v_{3}\right)\right\rangle$ and $f\left(v_{i}, v_{j}\right)=0 \Leftrightarrow f\left(\theta\left(v_{i}\right), \theta\left(v_{j}\right)\right)=0(i, j \in\{1,2,3\})$. So, $\mathcal{D}\left(\chi_{2}\right)=\mathcal{D}\left(\theta\left(\chi_{1}\right)\right)=\theta\left(\mathcal{D}\left(\chi_{1}\right)\right)$.

In the sequel of this section, we will suppose that $\operatorname{char}(\mathbb{F})=2$. For every hyperbolic basis $B=\left(e_{1}, f_{1}, e_{2}, f_{2}, e_{3}, f_{3}\right)$ of $(V, f)$ and every trivector

$$
\begin{gathered}
\chi=\lambda_{1} \cdot e_{1} \wedge e_{2} \wedge e_{3}+\mu_{1} \cdot f_{1} \wedge f_{2} \wedge f_{3}+\lambda_{2} \cdot e_{1} \wedge e_{2} \wedge f_{3}+\mu_{2} \cdot f_{1} \wedge f_{2} \wedge e_{3} \\
+\lambda_{3} \cdot e_{1} \wedge f_{2} \wedge e_{3}+\mu_{3} \cdot f_{1} \wedge e_{2} \wedge f_{3}+\lambda_{4} \cdot e_{1} \wedge f_{2} \wedge f_{3}+\mu_{4} \cdot f_{1} \wedge e_{2} \wedge e_{3} \\
+\lambda_{5} \cdot e_{1} \wedge e_{2} \wedge f_{2}+\mu_{5} \cdot f_{1} \wedge e_{3} \wedge f_{3}+\lambda_{6} \cdot e_{1} \wedge e_{3} \wedge f_{3}+\mu_{6} \cdot f_{1} \wedge e_{2} \wedge f_{2} \\
+\lambda_{7} \cdot e_{2} \wedge e_{1} \wedge f_{1}+\mu_{7} \cdot f_{2} \wedge e_{3} \wedge f_{3}+\lambda_{8} \cdot e_{2} \wedge e_{3} \wedge f_{3}+\mu_{8} \cdot f_{2} \wedge e_{1} \wedge f_{1} \\
+\lambda_{9} \cdot e_{3} \wedge e_{1} \wedge f_{1}+\mu_{9} \cdot f_{3} \wedge e_{2} \wedge f_{2}+\lambda_{10} \cdot e_{3} \wedge e_{2} \wedge f_{2}+\mu_{10} \cdot f_{3} \wedge e_{1} \wedge f_{1}
\end{gathered}
$$

of $V$, we define

$$
\eta_{B}(\chi):=\sum_{i=1}^{10} \lambda_{i} \mu_{i} .
$$

For every hyperbolic basis $B=\left(e_{1}, f_{1}, e_{2}, f_{2}, e_{3}, f_{3}\right)$ of $(V, f)$, let $\mathcal{B}_{B}$ denote the ordered basis $\left(e_{1} \wedge e_{2} \wedge e_{3}, f_{1} \wedge f_{2} \wedge f_{3}, e_{1} \wedge e_{2} \wedge f_{3}, f_{1} \wedge f_{2} \wedge e_{3}, e_{1} \wedge f_{2} \wedge e_{3}, f_{1} \wedge e_{2} \wedge f_{3}, e_{1} \wedge f_{2} \wedge\right.$ $f_{3}, f_{1} \wedge e_{2} \wedge e_{3}, e_{1} \wedge e_{2} \wedge f_{2}, f_{1} \wedge e_{3} \wedge f_{3}, e_{1} \wedge e_{3} \wedge f_{3}, f_{1} \wedge e_{2} \wedge f_{2}, e_{2} \wedge e_{1} \wedge f_{1}, f_{2} \wedge e_{3} \wedge f_{3}, e_{2} \wedge$ $\left.e_{3} \wedge f_{3}, f_{2} \wedge e_{1} \wedge f_{1}, e_{3} \wedge e_{1} \wedge f_{1}, f_{3} \wedge e_{2} \wedge f_{2}, e_{3} \wedge e_{2} \wedge f_{2}, f_{3} \wedge e_{1} \wedge f_{1}\right)$ of $\wedge^{3} V$

Proposition 3.10 Suppose $\operatorname{char}(\mathbb{F})=2$. Then for any two hyperbolic bases $B$ and $B^{\prime}$ of $(V, f)$, we have $\eta_{B}=\eta_{B^{\prime}}$.

Proof. In view of Lemma 3.2, it suffices to show that $\eta_{B}=\eta_{B^{\prime}}$ if $\left(B, B^{\prime}\right) \in \Omega_{1} \cup \Omega_{2} \cup$ $\cdots \cup \Omega_{5}$. This clearly holds if $\left(B, B^{\prime}\right) \in \Omega_{1}$. We will now also deal with the four remaining cases. Suppose $B=\left(e_{1}, f_{1}, e_{2}, f_{2}, e_{3}, f_{3}\right)$ and let $\chi$ be an arbitrary vector of $\bigwedge^{3} V$.

(1) Suppose $\left(B, B^{\prime}\right) \in \Omega_{2}$. Then $B^{\prime}=\left(\frac{e_{1}}{\lambda}, \lambda f_{1}, e_{2}, f_{2}, e_{3}, f_{3}\right)$ for some $\lambda \in \mathbb{F}^{*}$. If $\left(\lambda_{1}, \mu_{1}, \lambda_{2}, \mu_{2}, \ldots, \lambda_{10}, \mu_{10}\right)$ are the coordinates of $\chi$ with respect to the ordered basis $\mathcal{B}_{B^{\prime}}$, then $\left(\frac{\lambda_{1}}{\lambda}, \lambda \mu_{1}, \frac{\lambda_{2}}{\lambda}, \lambda \mu_{2}, \frac{\lambda_{3}}{\lambda}, \lambda \mu_{3}, \frac{\lambda_{4}}{\lambda}, \lambda \mu_{4}, \frac{\lambda_{5}}{\lambda}, \lambda \mu_{5}, \frac{\lambda_{6}}{\lambda}, \lambda \mu_{6}, \lambda_{7}, \mu_{7}, \lambda_{8}, \mu_{8}, \lambda_{9}, \mu_{9}, \lambda_{10}, \mu_{10}\right)$ are the coordinates of $\chi$ with respect to the ordered basis $\mathcal{B}_{B}$. So, we see that $\eta_{B^{\prime}}(\chi)=\eta_{B}(\chi)$.

(2) Suppose $\left(B, B^{\prime}\right) \in \Omega_{3}$. Then $B^{\prime}=\left(e_{1}+\lambda e_{2}, f_{1}, e_{2}, \lambda f_{1}+f_{2}, e_{3}, f_{3}\right)$ for some $\lambda \in \mathbb{F}$. If $\left(\lambda_{1}, \mu_{1}, \lambda_{2}, \mu_{2}, \ldots, \lambda_{10}, \mu_{10}\right)$ are the coordinates of $\chi$ with respect to the ordered basis $\mathcal{B}_{B^{\prime}}$, then $\left(\lambda_{1}, \mu_{1}, \lambda_{2}, \mu_{2}, \lambda_{3}, \mu_{3}+\lambda^{2} \lambda_{4}+\lambda \mu_{9}+\lambda \mu_{10}, \lambda_{4}, \lambda_{3} \lambda^{2}+\mu_{4}+\lambda \lambda_{9}+\lambda \lambda_{10}, \lambda_{5}, \mu_{5}+\right.$ $\left.\lambda \mu_{7}, \lambda_{6}, \mu_{6}+\lambda \mu_{8}, \lambda \lambda_{5}+\lambda_{7}, \mu_{7}, \lambda \lambda_{6}+\lambda_{8}, \mu_{8}, \lambda \lambda_{3}+\lambda_{9}, \mu_{9}+\lambda \lambda_{4}, \lambda \lambda_{3}+\lambda_{10}, \mu_{10}+\lambda \lambda_{4}\right)$ are the coordinates of $\chi$ with respect to the ordered basis $\mathcal{B}_{B}$. One verifies that $\eta_{B}(\chi)=\eta_{B^{\prime}}(\chi)$.

(3) Suppose $\left(B, B^{\prime}\right) \in \Omega_{4}$. Then $B^{\prime}=\left(e_{1}, f_{1}, e_{2}, f_{2}, e_{3}, f_{3}+\lambda e_{3}\right)$ for some $\lambda \in \mathbb{F}$. If $\left(\lambda_{1}, \mu_{1}, \lambda_{2}, \mu_{2}, \ldots, \lambda_{10}, \mu_{10}\right)$ are the coordinates of $\chi$ with respect to the ordered bases $\mathcal{B}_{B^{\prime}}$, then $\left(\lambda_{1}+\lambda \lambda_{2}, \mu_{1}, \lambda_{2}, \mu_{2}+\lambda \mu_{1}, \lambda_{3}+\lambda \lambda_{4}, \mu_{3}, \lambda_{4}, \mu_{4}+\lambda \mu_{3}, \lambda_{5}, \mu_{5}, \lambda_{6}, \mu_{6}, \lambda_{7}, \mu_{7}, \lambda_{8}, \mu_{8}, \lambda_{9}+\right.$ $\left.\lambda \mu_{10}, \mu_{9}, \lambda_{10}+\lambda \mu_{9}, \mu_{10}\right)$ are the coordinates of $\chi$ with respect to the ordered basis $\mathcal{B}_{B}$. One verifies that $\eta_{B}(\chi)=\eta_{B^{\prime}}(\chi)$. 
(4) Suppose $\left(B, B^{\prime}\right) \in \Omega_{5}$. Then $B^{\prime}=\left(e_{1}, f_{1}, e_{2}, f_{2}, e_{3}+\lambda f_{3}, f_{3}\right)$ for some $\lambda \in \mathbb{F}$. If $\left(\lambda_{1}, \mu_{1}, \lambda_{2}, \mu_{2}, \ldots, \lambda_{10}, \mu_{10}\right)$ are the coordinates of $\chi$ with respect to the ordered basis $\mathcal{B}_{B^{\prime}}$, then $\left(\lambda_{1}, \mu_{1}+\lambda \mu_{2}, \lambda \lambda_{1}+\lambda_{2}, \mu_{2}, \lambda_{3}, \mu_{3}+\lambda \mu_{4}, \lambda \lambda_{3}+\lambda_{4}, \mu_{4}, \lambda_{5}, \mu_{5}, \lambda_{6}, \mu_{6}, \lambda_{7}, \mu_{7}, \lambda_{8}, \mu_{8}, \lambda_{9}, \mu_{9}+\right.$ $\left.\lambda \lambda_{10}, \lambda_{10}, \mu_{10}+\lambda \lambda_{9}\right)$ are the coordinates of $\chi$ with respect to the ordered basis $\mathcal{B}_{B}$. One verifies that $\eta_{B}(\chi)=\eta_{B^{\prime}}(\chi)$.

Put $\eta:=\eta_{B}$ where $B$ is any hyperbolic basis of $(V, f)$.

Corollary 3.11 Suppose $\operatorname{char}(\mathbb{F})=2$. Then for every trivector $\chi$ and every $\theta \in S p(V, f)$, we have $\eta(\chi)=\eta(\theta(\chi))$.

Proof. Let $B$ be an arbitrary hyperbolic basis of $(V, f)$. Then we have $\eta(\theta(\chi))=$ $\eta_{\theta(B)}(\theta(\chi))=\eta_{B}(\chi)=\eta(\chi)$.

Lemma 3.12 Suppose $\operatorname{char}(\mathbb{F})=2$. Then $\eta\left(\chi+\chi^{\prime}\right)=\eta(\chi)$ for every $\chi \in W$ and every $\chi^{\prime} \in \widetilde{W}$.

Proof. Obviously, this holds if $\chi^{\prime}=0$. So, suppose $\chi^{\prime} \neq 0$ and put $\chi^{\prime}=\phi(v)$ where $v$ is some nonzero vector of $V$. There exists a hyperbolic basis $B=\left(e_{1}, f_{1}, e_{2}, f_{2}, e_{3}, f_{3}\right)$ of $(V, f)$ such that $v=e_{1}$. Let $\left(\lambda_{1}, \mu_{1}, \lambda_{2}, \mu_{2}, \ldots, \lambda_{10}, \mu_{10}\right)$ denote the coordinates of $\chi$ with respect to the ordered basis $\mathcal{B}_{B}$. Since $\chi \in W$, we have

$$
\lambda_{5}=\lambda_{6}, \mu_{5}=\mu_{6}, \lambda_{7}=\lambda_{8}, \mu_{7}=\mu_{8}, \lambda_{9}=\lambda_{10}, \mu_{9}=\mu_{10} .
$$

Now, the coordinates of $\chi+\phi(v)$ with respect to $\mathcal{B}_{B}$ are $\left(\lambda_{1}, \mu_{1}, \lambda_{2}, \mu_{2}, \lambda_{3}, \mu_{3}, \lambda_{4}, \mu_{4}, \lambda_{5}+\right.$ $\left.1, \mu_{5}, \lambda_{6}+1, \mu_{6}, \lambda_{7}, \mu_{7}, \lambda_{8}, \mu_{8}, \lambda_{9}, \mu_{9}, \lambda_{10}, \mu_{10}\right)$. So, we have $\eta\left(\chi+\chi^{\prime}\right)=\eta_{B}(\chi+\phi(v))=$ $\eta_{B}(\chi)+\left(\mu_{5}+\mu_{6}\right)=\eta_{B}(\chi)=\eta(\chi)$.

Corollary 3.11 and Lemma 3.12 then implies the following.

Corollary 3.13 Suppose $\operatorname{char}(\mathbb{F})=2$ and $\chi_{1}, \chi_{2}$ are two quasi-Sp $(V, f)$-equivalent trivectors of $W$. Then $\eta\left(\chi_{1}\right)=\eta\left(\chi_{2}\right)$.

Remark. The form $\eta$ defines a quadratic form on $\bigwedge^{3} V$, left invariant by $S p(V, f)$. Denote by $b: \bigwedge^{3} V \times \bigwedge^{3} V \rightarrow \mathbb{F} ;\left(\chi_{1}, \chi_{2}\right) \mapsto \eta\left(\chi_{1}+\chi_{2}\right)-\eta\left(\chi_{1}\right)-\eta\left(\chi_{2}\right)$ the nondegenerate alternating bilinear form associated to $\eta$. If $\eta^{\prime}$ and $b^{\prime}$ are the restrictions of $\eta$ and $b$ to $W$ and $W \times W$ respectively, then $\widetilde{W}$ is the radical of $b^{\prime}$ and is totally singular for $\eta^{\prime}$.

Also the following lemma will be useful in our classification.

Lemma 3.14 Suppose char $(\mathbb{F})=2$. Let $A$ and $A^{\prime}$ be two nonsingular $(3 \times 3)$-matrices over $\mathbb{F}$, and $\left(e_{1}, f_{1}, e_{2}, f_{2}, e_{3}, f_{3}\right)$ a hyperbolic basis of $(V, f)$. Put $\left[w_{1}, w_{2}, w_{3}\right]^{T}:=A \cdot\left[f_{1}, f_{2}, f_{3}\right]^{T}$ and $\left[w_{1}^{\prime}, w_{2}^{\prime}, w_{3}^{\prime}\right]^{T}:=A^{\prime} \cdot\left[f_{1}, f_{2}, f_{3}\right]^{T}$. If there exists $a \mu \in \mathbb{F}^{*}$ such that the matrices $\mu A$ and $A^{\prime}$ are pseudo-congruent, then the two trivectors $\chi=e_{1} \wedge e_{2} \wedge w_{3}+e_{2} \wedge e_{3} \wedge w_{1}+e_{3} \wedge e_{1} \wedge w_{2}$ and $\chi^{\prime}=e_{1} \wedge e_{2} \wedge w_{3}^{\prime}+e_{2} \wedge e_{3} \wedge w_{1}^{\prime}+e_{3} \wedge e_{1} \wedge w_{2}^{\prime}$ are quasi-Sp $(V, f)$-equivalent. 
Proof. Let $M$ be a nonsingular $(3 \times 3)$-matrix over $\mathbb{F}$ such that $\mu A-M A^{\prime} M^{T}$ is an alternating matrix. If we put $A^{\prime \prime}:=\frac{1}{\operatorname{det}(M)} \cdot\left(M A^{\prime} M^{T}\right)$ and $\left[w_{1}^{\prime \prime}, w_{2}^{\prime \prime}, w_{3}^{\prime \prime}\right]^{T}:=A^{\prime \prime} \cdot\left[f_{1}, f_{2}, f_{3}\right]^{T}$, then $\chi^{\prime \prime}:=e_{1} \wedge e_{2} \wedge w_{3}^{\prime \prime}+e_{2} \wedge e_{3} \wedge w_{1}^{\prime \prime}+e_{3} \wedge e_{1} \wedge w_{2}^{\prime \prime}$ is $S p(V, f)$-equivalent with $\chi^{\prime}$ by Lemma 3.5. Since $\frac{\mu}{\operatorname{det}(M)} \cdot A-A^{\prime \prime}$ is alternating, $\chi^{\prime \prime}$ is quasi- $S p(V, f)$-equivalent with $\frac{\mu}{\operatorname{det}(M)} \cdot \chi$. Now, $\chi$ and $\frac{\mu}{\operatorname{det}(M)} \cdot \chi$ are $S p(V, f)$-equivalent: if $\theta$ denotes the element of $S p(V, f)$ mapping $\left(e_{1}, f_{1}, e_{2}, f_{2}, e_{3}, f_{3}\right)$ to $\left(\frac{\mu e_{1}}{\operatorname{det}(M)}, \frac{\operatorname{det}(M) \cdot f_{1}}{\mu}, \frac{\mu e_{2}}{\operatorname{det}(M)}, \frac{\operatorname{det}(M) \cdot f_{2}}{\mu}, \frac{\mu e_{3}}{\operatorname{det}(M)}, \frac{\operatorname{det}(M) \cdot f_{3}}{\mu}\right)$, then $\theta$ maps the former trivector to the latter. We conclude that $\chi$ and $\chi^{\prime}$ are quasi- $S p(V, f)$-equivalent.

\section{Classification in the case $\operatorname{char}(\mathbb{F})=2$}

We continue with the notation introduced in Section 1. So, $V$ denotes a 6-dimensional vector space over a field $\mathbb{F}$ which is equipped with a nondegenerate alternating bilinear form $f$, and $\left(e_{1}, f_{1}, e_{2}, f_{2}, e_{3}, f_{3}\right)$ denotes a fixed hyperbolic basis of $(V, f)$. Throughout this section, we will suppose that $\operatorname{char}(\mathbb{F})=2$.

Our first goal is to prove Theorem 1.4. This will be achieved in a series of lemmas (4.1 till 4.14). Table 1 shows that the trivectors of $V$ whose type is either (A), (B), (C) or (D) can be divided into 19 families when one studies the $S p(V, f)$-equivalence between them. After studying the coarser relation of being quasi- $S p(V, f)$-equivalent, it will turn out that a description using only nine families is already sufficient, see Corollary 4.15 (which is precisely Theorem 1.4). Our next goal will be to show that none of these nine families is superfluous in the description. In Lemma 4.23 (which is precisely the first claim of Theorem 1.5), we will indeed show that trivectors belonging to distinct families (among these nine) can never be quasi- $S p(V, f)$-equivalent. This lemma implies that it suffices to study quasi- $S p(V, f)$-equivalence between trivectors belonging the same family. This will be done in the six lemmas at the end of this section, and will prove the remaining claims of Theorem 1.5.

Lemma 4.1 The trivector $\chi_{B 1}$ is quasi-Sp $(V, f)$-equivalent with the trivector $\chi_{D 4}(1,1)$.

Proof. Let $\theta$ be the element of $S p(V, f)$ mapping the hyperbolic basis $\left(e_{1}, f_{1}, e_{2}, f_{2}, e_{3}, f_{3}\right)$ of $(V, f)$ to the hyperbolic basis $\left(e_{2}+e_{3}, f_{1}+f_{3}, e_{1}+e_{2}+e_{3}, f_{1}, f_{2}+f_{3}, e_{2}\right)$ of $(V, f)$. Then $\theta$ maps the trivector $\chi_{B 1}=e_{1} \wedge e_{2} \wedge e_{3}+e_{1} \wedge f_{1} \wedge f_{3}$ to the trivector

$$
\begin{aligned}
& \left(e_{2}+e_{3}\right) \wedge\left(e_{1}+e_{2}+e_{3}\right) \wedge\left(f_{2}+f_{3}\right)+\left(e_{2}+e_{3}\right) \wedge\left(f_{1}+f_{3}\right) \wedge e_{2} \\
= & e_{1} \wedge\left(e_{2}+e_{3}\right) \wedge\left(f_{2}+f_{3}\right)+e_{2} \wedge e_{3} \wedge\left(f_{1}+f_{3}\right) \\
= & e_{1} \wedge e_{2} \wedge f_{3}+e_{2} \wedge e_{3} \wedge\left(f_{1}+f_{3}\right)+e_{1} \wedge e_{3} \wedge f_{2}+e_{1} \wedge\left(e_{2} \wedge f_{2}+e_{3} \wedge f_{3}\right) \\
= & \chi_{D 4}(1,1)+e_{1} \wedge\left(e_{2} \wedge f_{2}+e_{3} \wedge f_{3}\right) .
\end{aligned}
$$

It follows that $\chi_{B 1}$ is quasi- $S p(V, f)$-equivalent with $\chi_{D 4}(1,1)$.

Lemma 4.2 The trivector $\chi_{B 2}$ is quasi-Sp $(V, f)$-equivalent with $\chi_{A 2}$. 
Proof. The trivector $\chi_{B 2}=e_{1} \wedge e_{2} \wedge f_{2}+e_{1} \wedge f_{1} \wedge e_{3}$ is quasi- $S p(V, f)$-equivalent with $e_{1} \wedge e_{2} \wedge f_{2}+e_{1} \wedge f_{1} \wedge e_{3}+e_{3} \wedge\left(e_{1} \wedge f_{1}+e_{2} \wedge f_{2}\right)=\left(e_{1}+e_{3}\right) \wedge e_{2} \wedge f_{2}$ and the latter trivector is $S p(V, f)$-equivalent with $\chi_{A 2}$.

The following result is obvious.

Lemma 4.3 The trivector $\chi_{B 3}$ is quasi-Sp $(V, f)$-equivalent with the 0-vector of $\bigwedge^{3} V$. In general, the trivectors quasi-Sp $(V, f)$-equivalent with the 0-vector of $\wedge^{3} V$ are precisely the vectors of $\widetilde{W}$.

Lemma 4.4 For every $\lambda \in \mathbb{F}^{*}$, the trivector $\chi_{C 2}(\lambda)$ is quasi-Sp $(V, f)$-equivalent with the trivector $\chi_{D 2}\left(\lambda^{2}\right)$.

Proof. The trivector $\chi_{C 2}(\lambda)=f_{1} \wedge\left(e_{2}+e_{3}\right) \wedge\left(f_{2}-f_{3}\right)+\lambda \cdot e_{1} \wedge e_{2} \wedge f_{2}$ is quasi- $S p(V, f)$ equivalent with $f_{1} \wedge e_{2} \wedge f_{3}+f_{1} \wedge e_{3} \wedge f_{2}+\lambda \cdot e_{1} \wedge e_{2} \wedge f_{2}=\lambda^{2} \cdot e_{2}^{\prime} \wedge e_{1}^{\prime} \wedge f_{3}^{\prime}+f_{1}^{\prime} \wedge e_{2}^{\prime} \wedge e_{3}^{\prime}+e_{1}^{\prime} \wedge f_{1}^{\prime} \wedge f_{2}^{\prime}$, where $\left(e_{1}^{\prime}, f_{1}^{\prime}, e_{2}^{\prime}, f_{2}^{\prime}, e_{3}^{\prime}, f_{3}^{\prime}\right)$ is the hyperbolic basis $\left(e_{2}, f_{2}, \frac{f_{1}}{\lambda}, \lambda e_{1}, \lambda e_{3}, \frac{f_{3}}{\lambda}\right)$ of $(V, f)$. So, $\chi_{C 2}(\lambda)$ is quasi- $S p(V, f)$-equivalent with $\chi_{D 2}\left(\lambda^{2}\right)$.

Lemma 4.5 For every $\lambda \in \mathbb{F}^{*}$, the trivector $\chi_{C 3}(\lambda)$ is quasi-Sp $(V, f)$-equivalent with $\chi_{A 2}$.

Proof. The trivector $\chi_{C 3}(\lambda)=e_{1} \wedge e_{2} \wedge f_{2}+\lambda \cdot f_{1} \wedge e_{3} \wedge f_{3}$ is quasi- $S p(V, f)$-equivalent with $e_{1} \wedge e_{3} \wedge f_{3}+\lambda \cdot f_{1} \wedge e_{3} \wedge f_{3}=\left(e_{1}+\lambda f_{1}\right) \wedge e_{3} \wedge f_{3}$, and the latter trivector is $S p(V, f)$-equivalent with $\chi_{A 2}$.

Lemma 4.6 For every $\lambda \in \mathbb{F}^{*}$, the trivector $\chi_{C 4}(\lambda)$ is quasi-Sp $(V, f)$-equivalent with $\chi_{B 1}$ and hence also with $\chi_{D 4}(1,1)$.

Proof. The trivector $\chi_{C 4}(\lambda)=f_{1} \wedge e_{3} \wedge\left(e_{2}+f_{3}\right)+\lambda \cdot e_{1} \wedge e_{2} \wedge f_{2}$ is quasi- $S p(V, f)$-equivalent with $f_{1} \wedge e_{3} \wedge\left(e_{2}+f_{3}\right)+\lambda \cdot e_{1} \wedge e_{3} \wedge f_{3}=e_{3} \wedge e_{2} \wedge f_{1}+e_{3} \wedge f_{3} \wedge\left(f_{1}+\lambda e_{1}\right)=e_{1}^{\prime} \wedge e_{2}^{\prime} \wedge e_{3}^{\prime}+e_{1}^{\prime} \wedge f_{1}^{\prime} \wedge f_{3}^{\prime}$, where $\left(e_{1}^{\prime}, f_{1}^{\prime}, e_{2}^{\prime}, f_{2}^{\prime}, e_{3}^{\prime}, f_{3}^{\prime}\right)$ is the hyperbolic basis $\left(e_{3}, f_{3}, \lambda e_{2}, \frac{f_{2}}{\lambda}, \frac{f_{1}}{\lambda}, f_{1}+\lambda e_{1}\right)$ of $(V, f)$. So, $\chi_{C 4}(\lambda)$ is quasi- $S p(V, f)$-equivalent with $\chi_{B 1}$ and hence also with $\chi_{D 4}(1,1)$ by Lemma 4.1 .

Lemma 4.7 For every $\lambda \in \mathbb{F}^{*}$, the trivector $\chi_{C 5}(\lambda)$ is quasi-Sp $(V, f)$-equivalent with $\chi_{C 2}(\lambda)$ and hence also with $\chi_{D 2}\left(\lambda^{2}\right)$.

Proof. Let $\left(e_{1}^{\prime}, f_{1}^{\prime}, e_{2}^{\prime}, f_{2}^{\prime}, e_{3}^{\prime}, f_{3}^{\prime}\right)$ be the hyperbolic basis $\left(\frac{e_{1}}{\lambda}+e_{2}, \lambda f_{1}, e_{3}, \lambda f_{1}+f_{2}+f_{3}, e_{2}+\right.$ $\left.e_{3}, \lambda e_{2}+\lambda e_{3}+\lambda f_{1}+f_{2}\right)$ of $(V, f)$. Then $f_{1}^{\prime} \wedge\left(e_{2}^{\prime}+e_{3}^{\prime}\right) \wedge\left(f_{2}^{\prime}-f_{3}^{\prime}\right)+\lambda \cdot e_{1}^{\prime} \wedge e_{2}^{\prime} \wedge f_{2}^{\prime}$ is $S p(V, f)$-equivalent with $\chi_{C 2}(\lambda)$ and equal to

$e_{1} \wedge e_{3} \wedge\left(f_{3}+f_{2}\right)+\lambda \cdot e_{2} \wedge f_{3} \wedge\left(f_{1}+e_{3}\right)+\lambda \cdot e_{3} \wedge\left(e_{1} \wedge f_{1}+e_{2} \wedge f_{2}\right)=\chi_{C 5}(\lambda)+\phi\left(\lambda e_{3}\right)$.

So, $\chi_{C 5}(\lambda)$ is quasi- $S p(V, f)$-equivalent with $\chi_{C 2}(\lambda)$ and hence also with $\chi_{D 2}\left(\lambda^{2}\right)$ by Lemma 4.4 . 
Lemma 4.8 For every $\lambda \in \mathbb{F}^{*}$ and every $\epsilon \in \mathbb{F} \backslash\{0,-1\}$, the trivector $\chi_{C 6}(\lambda, \epsilon)$ is quasi$\operatorname{Sp}(V, f)$-equivalent with $\chi_{D 2}\left(\lambda^{2} \epsilon\right)$.

Proof. The trivector $\chi_{C 6}(\lambda, \epsilon)=f_{1} \wedge\left(e_{2}+e_{3}\right) \wedge\left(f_{2}+\epsilon f_{3}\right)+\lambda \cdot e_{1} \wedge e_{2} \wedge f_{2}=f_{1} \wedge e_{2} \wedge f_{2}+$ $\epsilon \cdot f_{1} \wedge e_{2} \wedge f_{3}+f_{1} \wedge e_{3} \wedge f_{2}+\epsilon \cdot f_{1} \wedge e_{3} \wedge f_{3}+\lambda \cdot e_{1} \wedge e_{2} \wedge f_{2}$ is quasi- $S p(V, f)$-equivalent with $\left((1+\epsilon) f_{1}+\lambda e_{1}\right) \wedge e_{2} \wedge f_{2}+f_{1} \wedge e_{2} \wedge\left(\epsilon f_{3}\right)+f_{1} \wedge f_{2} \wedge e_{3}=\lambda^{2} \epsilon \cdot e_{1}^{\prime} \wedge e_{2}^{\prime} \wedge f_{3}^{\prime}+e_{2}^{\prime} \wedge f_{1}^{\prime} \wedge e_{3}^{\prime}+f_{1}^{\prime} \wedge e_{1}^{\prime} \wedge f_{2}^{\prime}$, where $\left(e_{1}^{\prime}, f_{1}^{\prime}, e_{2}^{\prime}, f_{2}^{\prime}, e_{3}^{\prime}, f_{3}^{\prime}\right)$ is the hyperbolic basis $\left(f_{2}, e_{2}, \frac{f_{1}}{\lambda},(1+\epsilon) f_{1}+\lambda e_{1}, \lambda \epsilon f_{3}, \frac{e_{3}}{\lambda \epsilon}\right)$ of $(V, f)$. So, $\chi_{C 6}(\lambda, \epsilon)$ is quasi- $S p(V, f)$-equivalent with $\chi_{D 2}\left(\lambda^{2} \epsilon\right)$.

Lemma 4.9 The trivector $\chi_{D 1}$ is quasi-Sp $(V, f)$-equivalent with $\chi_{B 1}$ and hence also with $\chi_{D 4}(1,1)$.

Proof. The trivector $\chi_{D 1}=e_{1} \wedge e_{2} \wedge f_{2}+e_{2} \wedge f_{1} \wedge e_{3}+f_{1} \wedge e_{1} \wedge f_{3}$ is quasi- $S p(V, f)$ equivalent with $e_{1} \wedge e_{2} \wedge f_{2}+e_{2} \wedge f_{1} \wedge e_{3}+e_{2} \wedge f_{2} \wedge f_{3}=e_{1}^{\prime} \wedge e_{2}^{\prime} \wedge e_{3}^{\prime}+e_{1}^{\prime} \wedge f_{1}^{\prime} \wedge f_{3}^{\prime}$, where $\left(e_{1}^{\prime}, f_{1}^{\prime}, e_{2}^{\prime}, f_{2}^{\prime}, e_{3}^{\prime}, f_{3}^{\prime}\right)$ is the hyperbolic basis $\left(e_{2}, f_{2}, f_{1}+e_{3}, e_{1}, e_{3}, e_{1}+f_{3}\right)$ of $(V, f)$. So, $\chi_{D 1}$ is quasi- $S p(V, f)$-equivalent with $\chi_{B 1}$ and hence also with $\chi_{D 4}(1,1)$ by Lemma 4.1.

Lemma 4.10 For every $\lambda \in \mathbb{F}^{*}$, the trivector $\chi_{D 5}(\lambda)$ is quasi-Sp $(V, f)$-equivalent with $\chi_{B 5}(\lambda)$.

Proof. Let $\theta$ be the unique element of $S p(V, f)$ mapping the hyperbolic basis $\left(e_{1}, f_{1}, e_{2}, f_{2}\right.$, $\left.e_{3}, f_{3}\right)$ of $(V, f)$ to the hyperbolic basis $\left(e_{2}+e_{3}, f_{2}, e_{3}, f_{1}+f_{2}+f_{3}, e_{1}+e_{3}, f_{1}\right)$ of $(V, f)$. Then $\theta$ maps $\chi_{B 5}(\lambda)=\lambda \cdot e_{1} \wedge e_{2} \wedge f_{2}+e_{1} \wedge\left(e_{2}-e_{3}\right) \wedge\left(f_{2}+f_{3}\right)$ to

$$
\begin{aligned}
& \lambda \cdot\left(e_{2}+e_{3}\right) \wedge e_{3} \wedge\left(f_{1}+f_{2}+f_{3}\right)+\left(e_{2}+e_{3}\right) \wedge e_{1} \wedge\left(f_{2}+f_{3}\right) \\
= & \lambda \cdot e_{2} \wedge e_{3} \wedge\left(f_{1}+f_{2}+f_{3}\right)+e_{1} \wedge e_{2} \wedge f_{3}+e_{1} \wedge e_{3} \wedge f_{2}+e_{1} \wedge\left(e_{2} \wedge f_{2}+e_{3} \wedge f_{3}\right) \\
= & \chi_{D 5}(\lambda)+e_{1} \wedge\left(e_{2} \wedge f_{2}+e_{3} \wedge f_{3}\right) .
\end{aligned}
$$

So, the trivectors $\chi_{D 5}(\lambda)$ and $\chi_{B 5}(\lambda)$ are indeed quasi- $S p(V, f)$-equivalent.

Lemma 4.11 If $|\mathbb{F}|=2$, then the trivector $\chi_{D 7}$ is quasi-Sp $(V, f)$-equivalent with the trivector $\chi_{A 2}$.

Proof. The trivector $\chi_{D 7}=e_{1} \wedge e_{2} \wedge f_{2}+e_{2} \wedge e_{3} \wedge\left(f_{1}+f_{3}\right)+e_{3} \wedge e_{1} \wedge f_{3}$ is quasi- $S p(V, f)$ equivalent with the trivector $e_{2} \wedge e_{3} \wedge\left(f_{1}+f_{3}\right)$, which is itself $S p(V, f)$-equivalent with $\chi_{A 2}$.

Lemma 4.12 If $\lambda$ is a square in $\mathbb{F}^{*}$, then the trivector $\chi_{B 4}(\lambda)$ is quasi-Sp $(V, f)$-equivalent with $\chi_{A 1}$.

Proof. Suppose $\lambda=\mu^{2}$ where $\mu \in \mathbb{F}^{*}$. Then $\chi_{B 4}(\lambda)=e_{1} \wedge e_{2} \wedge e_{3}+\lambda \cdot e_{1} \wedge f_{2} \wedge f_{3}=$ $e_{1} \wedge\left(e_{2}+\mu f_{3}\right) \wedge\left(e_{3}+\mu f_{2}\right)+\mu \cdot e_{1} \wedge\left(e_{2} \wedge f_{2}+e_{3} \wedge f_{3}\right)$ is quasi- $S p(V, f)$-equivalent with the trivector $e_{1} \wedge\left(e_{2}+\mu f_{3}\right) \wedge\left(e_{3}+\mu f_{2}\right)$ which itself is $S p(V, f)$-equivalent with $\chi_{A 1}$.

Lemma 4.13 Let $\lambda \in \mathbb{F}^{*}$. If the equation $X^{2}+\lambda X+1$ has a solution for $X \in \mathbb{F}$, then $\chi_{B 5}(\lambda)$ is quasi-Sp $(V, f)$-equivalent with $\chi_{A 2}$. 
Proof. Suppose $\mu^{2}+\mu \lambda+1=0$ for some $\mu \in \mathbb{F}$. Then $\mu \neq 0$ and $\lambda=\mu+\frac{1}{\mu}$. Now, $\chi_{B 5}(\lambda)=\left(\mu+\frac{1}{\mu}\right) \cdot e_{1} \wedge e_{2} \wedge f_{2}+e_{1} \wedge\left(e_{2}+e_{3}\right) \wedge\left(f_{2}+f_{3}\right)=\left(\lambda e_{1}\right) \wedge \frac{e_{2}+\mu e_{3}}{\lambda} \wedge\left(\frac{1}{\mu} f_{2}+f_{3}\right)+$ $(\mu+1) \cdot e_{1} \wedge\left(e_{2} \wedge f_{2}+e_{3} \wedge f_{3}\right)$ is quasi- $S p(V, f)$-equivalent with $\left(\lambda e_{1}\right) \wedge \frac{e_{2}+\mu e_{3}}{\lambda} \wedge\left(\frac{1}{\mu} f_{2}+f_{3}\right)$ which is a trivector of Type (A2) since $f\left(\frac{e_{2}+\mu e_{3}}{\lambda}, \frac{f_{2}}{\mu}+f_{3}\right)=1$.

Lemma 4.14 Let $\lambda_{1}, \lambda_{2} \in \mathbb{F}^{*}$. If the equation $\lambda_{1} X^{2}+\lambda_{2} Y^{2}+Z^{2}=0$ has a nonzero solution for $(X, Y, Z) \in \mathbb{F}^{3}$, then the trivector $\chi_{D 3}\left(\lambda_{1}, \lambda_{2}\right)$ is quasi-Sp $(V, f)$-equivalent with $\chi_{B 4}\left(\lambda_{1}\right)$ or $\chi_{B 4}\left(\lambda_{2}\right)$.

Proof. Let $(\alpha, \beta, \gamma) \in \mathbb{F}^{3} \backslash\{(0,0,0)\}$ such that $\lambda_{1} \alpha^{2}+\lambda_{2} \beta^{2}+\gamma^{2}=0$. It is impossible that $\alpha=\beta=0$. So, $\alpha \neq 0$ or $\beta \neq 0$.

Suppose $\alpha \neq 0$. Then $\chi_{D 3}\left(\lambda_{1}, \lambda_{2}\right)=e_{1} \wedge e_{2} \wedge f_{3}+\lambda_{1} \cdot e_{2} \wedge e_{3} \wedge f_{1}+\lambda_{2} \cdot e_{3} \wedge e_{1} \wedge f_{2}$ is equal to

$$
\frac{e_{1}^{\prime}}{\alpha} \wedge\left(e_{2}^{\prime} \wedge e_{3}^{\prime}+\lambda_{2} \cdot f_{2}^{\prime} \wedge f_{3}^{\prime}\right)+\frac{\gamma}{\alpha} \cdot e_{2}^{\prime} \wedge\left(e_{1}^{\prime} \wedge f_{1}^{\prime}+e_{3}^{\prime} \wedge f_{3}^{\prime}\right)+\frac{\lambda_{2} \beta}{\alpha} \cdot f_{3}^{\prime} \wedge\left(e_{1}^{\prime} \wedge f_{1}^{\prime}+e_{2}^{\prime} \wedge f_{2}^{\prime}\right)
$$

where $\left(e_{1}^{\prime}, f_{1}^{\prime}, e_{2}^{\prime}, f_{2}^{\prime}, e_{3}^{\prime}, f_{3}^{\prime}\right)$ is the hyperbolic basis $\left(\alpha e_{1}+\beta e_{2}+\gamma e_{3}, \frac{f_{1}}{\alpha}, e_{2}, f_{2}+\frac{\beta}{\alpha} f_{1}, f_{3}+\right.$ $\left.\frac{\gamma}{\alpha} f_{1}, e_{3}\right)$ of $(V, f)$. So, $\chi_{D 3}\left(\lambda_{1}, \lambda_{2}\right)$ is quasi- $S p(V, f)$-equivalent with $\frac{e_{1}^{\prime}}{\alpha} \wedge\left(e_{2}^{\prime} \wedge e_{3}^{\prime}+\lambda_{2} \cdot f_{2}^{\prime} \wedge f_{3}^{\prime}\right)$ which itself is $S p(V, f)$-equivalent with $\chi_{B 4}\left(\lambda_{2}\right)$.

So, if $\alpha \neq 0$, then we know that $\chi_{D 3}\left(\lambda_{1}, \lambda_{2}\right)$ is quasi- $S p(V, f)$-equivalent with $\chi_{B 4}\left(\lambda_{2}\right)$. If $\beta \neq 0$, then by reasons of symmetry, we know that $\chi_{D 3}\left(\lambda_{1}, \lambda_{2}\right)$ is quasi- $S p(V, f)$ equivalent with $\chi_{B 4}\left(\lambda_{1}\right)$.

The following corollary, which is precisely Theorem 1.4, is a consequence of Lemmas 4.1 -4.14 .

Corollary 4.15 Let $\chi$ be a trivector of $V$ quasi-Sp $(V, f)$-equivalent with a trivector of Type (A), (B), (C) or (D). Then $\chi$ is quasi-Sp $(V, f)$-equivalent with (at least) one of the following trivectors:

$\left(Q 1^{\prime}\right)$ the zero vector of $\bigwedge^{3} V$;

$\left(Q 2^{\prime}\right) \chi_{A 1}$;

$\left(Q 3^{\prime}\right) \chi_{A 2}$;

$\left(Q 4^{\prime}\right) \chi_{B 4}(\lambda)$ for some nonsquare $\lambda$ of $\mathbb{F}$;

$\left(Q 5^{\prime}\right) \chi_{B 5}(\lambda)$ for some $\lambda \in \mathbb{F}$ such that the polynomial $X^{2}+\lambda X+1 \in \mathbb{F}[X]$ is irreducible;

$\left(Q 6^{\prime}\right) \chi_{C 1}(\lambda)$ for some $\lambda \in \mathbb{F}^{*}$;

$\left(Q 7^{\prime}\right) \chi_{D 2}(\lambda)$ for some $\lambda \in \mathbb{F}^{*}$;

$\left(Q 8^{\prime}\right) \chi_{D 3}\left(\lambda_{1}, \lambda_{2}\right)$ for some $\lambda_{1}, \lambda_{2} \in \mathbb{F}^{*}$ such that the equation $\lambda_{1} X^{2}+\lambda_{2} Y^{2}+Z^{2}=0$ has no solutions for $(X, Y, Z) \in \mathbb{F}^{3} \backslash\{(0,0,0)\}$;

$\left(Q 9^{\prime}\right) \chi_{D 4}\left(\lambda_{1}, \lambda_{2}\right)$ for some $\lambda_{1}, \lambda_{2} \in \mathbb{F}^{*}$.

Our next goal will be to show that trivectors belonging to distinct families (as occurring in Corollary 4.15) can never be quasi- $S p(V, f)$-equivalent. 
Lemma 4.16 Let $\chi_{1} \in W$ and $\chi_{2} \notin W$. Then $\chi_{1}$ and $\chi_{2}$ are not quasi-Sp $(V, f)$ equivalent.

Proof. Suppose $\chi_{1}$ and $\chi_{2}$ are quasi- $S p(V, f)$-equivalent. Then there exists a $\theta \in S p(V, f)$ and a $\chi \in \widetilde{W}$ such that $\chi_{2}=\theta\left(\chi_{1}\right)+\chi$. Since $\theta\left(\chi_{1}\right) \in W$ and $\chi \in \widetilde{W} \subset W$, we must have that $\chi_{2} \in W$, a contradiction.

Corollary 4.17 Let $i \in\{1,2,4,6,8\}$ and $j \in\{3,5,7,9\}$. Then no trivector of Type $\left(Q i^{\prime}\right)$ is quasi-Sp $(V, f)$-equivalent with a trivector of Type $\left(Q j^{\prime}\right)$.

Proof. This follows from Lemma 4.16, taking into account that a trivector of Type (Q $\left.i^{\prime}\right)$ belongs to $W$, while a trivector of Type $\left(\mathrm{Q} j^{\prime}\right)$ does not belong to $W$.

Lemma 4.18 Let $i \in\{2,3, \ldots, 9\}$. Then no trivector of Type $\left(Q i^{\prime}\right)$ is quasi-Sp $(V, f)$ equivalent with the zero vector.

Proof. The nonzero trivectors quasi- $S p(V, f)$-equivalent with the zero trivector are precisely the trivectors $S p(V, f)$-equivalent with $\chi_{B 3}$ and none of these trivectors is of Type $\left(\mathrm{Q} i^{\prime}\right)$.

Recall that $\overline{\mathbb{F}}$ is a fixed algebraic closure of $\mathbb{F}$. For every field $\mathbb{K}$ satisfying $\mathbb{F} \subseteq \mathbb{K} \subseteq \overline{\mathbb{F}}$, the vector space $V$ naturally embeds into a $\mathbb{K}$-vector space $V_{\mathbb{K}}$ by allowing the coordinates (with respect to $\left\{e_{1}, f_{1}, \ldots, e_{3}, f_{3}\right\}$ ) to be elements of $\mathbb{K}$. The nondegenerate alternating bilinear form $f$ naturally extends to a nondegenerate alternating bilinear form $f_{\mathbb{K}}$ of $V_{\mathbb{K}}$. Every trivector of $V$ can also be regarded as a trivector of $V_{\mathbb{K}}$. For every trivector $\chi \in \bigwedge^{3} V_{\mathbb{K}}$, we can define a set $\mathcal{D}_{\mathbb{K}}(\chi) \subseteq V_{\mathbb{K}}$ in a similar way as in Section 3. Notice that we have used a subindex to indicate the underlying field in order to avoid confusion. Indeed, if $\chi \in \bigwedge^{3} V$, then $\chi$ can also be regarded as an element of $\bigwedge^{3} V_{\mathbb{K}}$ and the sets $\mathcal{D}(\chi)$ and $\mathcal{D}_{\mathbb{K}}(\chi)$ need not to be equal.

Lemma 4.19 Let $\mathbb{K}$ be a field such that $\mathbb{F} \subseteq \mathbb{K} \subseteq \overline{\mathbb{F}}$. Suppose $\chi=e_{1} \wedge e_{2} \wedge w_{3}+e_{2} \wedge$ $e_{3} \wedge w_{1}+e_{3} \wedge e_{1} \wedge w_{2}$, where $\left[w_{1}, w_{2}, w_{3}\right]^{T}=A \cdot\left[f_{1}, f_{2}, f_{3}\right]^{T}$ for some $(3 \times 3)$-matrix $A=\left(a_{i j}\right)_{1 \leq i, j \leq 3}$ over $\mathbb{K}$. Let $\left(\alpha_{1}, \alpha_{2}, \alpha_{3}\right) \in \mathbb{K}^{3} \backslash\{(0,0,0)\}$, and put $v=\alpha_{1} e_{1}+\alpha_{2} e_{2}+\alpha_{3} e_{3}$. Then the following are equivalent:

(1) $v \in \mathcal{D}_{\mathbb{K}}(\chi)$;

(2) $\sum_{1 \leq i, j \leq 3} a_{i j} \alpha_{i} \alpha_{j}=0$;

(3) there exist vectors $v^{\prime}, v^{\prime \prime} \in V_{\mathbb{K}}$ such that $\left\langle v, v^{\prime}, v^{\prime \prime}\right\rangle_{\mathbb{K}}$ is a 3-space totally isotropic for $f_{\mathbb{K}},\left\langle v, v^{\prime}, v^{\prime \prime}\right\rangle_{\mathbb{K}} \cap\left\langle e_{1}, e_{2}, e_{3}\right\rangle_{\mathbb{K}}=\langle v\rangle_{\mathbb{K}}$ and $\chi \wedge v \wedge v^{\prime} \wedge v^{\prime \prime}=0$.

Proof. Put $v_{1}^{(1)}=e_{1}, v_{2}^{(1)}=e_{2}$ and $v_{3}^{(1)}=e_{3}$, and let $v_{i}^{(j)}$ with $i \in\{1,2,3\}$ and $j \in\{2,3,4,5\}$ be vectors of $V_{\mathbb{K}}$ as in Lemma 3.7. By Property (v) of Lemma 3.7, $\chi \wedge$ $v_{1}^{(j)} \wedge v_{2}^{(j)} \wedge v_{3}^{(j)}=0$ for every $j \in\{1,2,3,4\}$. So, we have that $\alpha_{1} e_{1}+\alpha_{2} e_{2}+\alpha_{3} e_{3} \in \mathcal{D}_{\mathbb{K}}(\chi)$ if and only if $\chi \wedge v_{1}^{(5)} \wedge v_{2}^{(5)} \wedge v_{3}^{(5)}=0$. The equivalence of (1) and (3) follows. 
If $\alpha_{2} \neq 0$, then we can take $v_{1}^{(5)}=\alpha_{1} e_{1}+\alpha_{2} e_{2}+\alpha_{3} e_{3}, v_{2}^{(5)}=\alpha_{2} f_{1}+\alpha_{1} f_{2}$ and $v_{3}^{(5)}=$ $\alpha_{3} f_{2}+\alpha_{2} f_{3}$. In this case, we put $\chi^{\prime}:=\frac{1}{\alpha_{2}} \cdot\left(\alpha_{1} e_{1}+\alpha_{2} e_{2}+\alpha_{3} e_{3}\right) \wedge\left(\alpha_{2} f_{1}+\alpha_{1} f_{2}\right) \wedge\left(\alpha_{3} f_{2}+\alpha_{2} f_{3}\right)$.

If $\alpha_{2}=0$, then we can take $v_{1}^{(5)}=\alpha_{1} e_{1}+\alpha_{3} e_{3}, v_{2}^{(5)}=f_{2}$ and $v_{3}^{(5)}=\alpha_{3} f_{1}+\alpha_{1} f_{3}$. In this case, we put $\chi^{\prime}:=\left(\alpha_{1} e_{1}+\alpha_{3} e_{3}\right) \wedge f_{2} \wedge\left(\alpha_{3} f_{1}+\alpha_{1} f_{3}\right)$.

In any case, we have $\chi^{\prime}=\alpha_{1} \alpha_{3} \cdot e_{1} \wedge f_{1} \wedge f_{2}+\alpha_{1} \alpha_{2} \cdot e_{1} \wedge f_{1} \wedge f_{3}+\alpha_{1}^{2} \cdot e_{1} \wedge f_{2} \wedge f_{3}+\alpha_{2} \alpha_{3} \cdot e_{2} \wedge$ $f_{1} \wedge f_{2}+\alpha_{2}^{2} \cdot e_{2} \wedge f_{1} \wedge f_{3}+\alpha_{1} \alpha_{2} \cdot e_{2} \wedge f_{2} \wedge f_{3}+\alpha_{3}^{2} \cdot e_{3} \wedge f_{1} \wedge f_{2}+\alpha_{2} \alpha_{3} \cdot e_{3} \wedge f_{1} \wedge f_{3}+\alpha_{1} \alpha_{3} \cdot e_{3} \wedge f_{2} \wedge f_{3}$. Now, $\alpha_{1} e_{1}+\alpha_{2} e_{2}+\alpha_{3} e_{3} \in \mathcal{D}_{\mathbb{K}}(\chi)$ if and only if $\chi \wedge \chi^{\prime}=0$. This happens precisely when $\sum_{1 \leq i, j \leq 3} a_{i j} \alpha_{i} \alpha_{j}=0$.

Lemma 4.20 (1) We have $\mathcal{D}\left(\chi_{A 1}\right)=\left\langle e_{1}, e_{2}, e_{3}\right\rangle$.

(2) For every nonsquare $\lambda$ of $\mathbb{F}$, we have $\mathcal{D}\left(\chi_{B 4}(\lambda)\right)=\left\langle e_{1}\right\rangle$.

(3) For every $\lambda \in \mathbb{F}^{*}$, we have $\mathcal{D}\left(\chi_{C 1}(\lambda)\right)=\mathcal{D}_{\overline{\mathbb{F}}}\left(\chi_{C 1}(\lambda)\right)=\{o\}$.

(4) For all $\lambda_{1}, \lambda_{2} \in \mathbb{F}^{*}$ such that the equation $\lambda_{1} X^{2}+\lambda_{2} Y^{2}+Z^{2}=0$ has no solution for $(X, Y, Z) \in \mathbb{F}^{3} \backslash\{(0,0,0)\}$, we have $\mathcal{D}\left(\chi_{D 3}\left(\lambda_{1}, \lambda_{2}\right)\right)=\{o\}$ and $\mathcal{D}_{\overline{\mathbb{F}}}\left(\chi_{D 3}\left(\lambda_{1}, \lambda_{2}\right)\right) \neq$ $\{o\}$.

Proof. In [17], a method was described to determine the sets $\mathcal{D}(\chi)$ for trivectors $\chi$ of $V$. In Section 3 of that paper, this method was applied to some particular cases. These cases include all trivectors of Type A1, B4 and C1. Claims (1), (2) and (3) of the lemma follow from that treatment. We will now also prove Claim (4).

Put $\chi:=\chi_{D 3}\left(\lambda_{1}, \lambda_{2}\right)$. By Lemma 4.19, the elements of $\mathcal{D}(\chi)$ and $\mathcal{D}_{\overline{\mathbb{F}}}(\chi)$ of the form $\alpha_{1} e_{1}+\alpha_{2} e_{2}+\alpha_{3} e_{3}$ are determined by the equation $\lambda_{1} \alpha_{1}^{2}+\lambda_{2} \alpha_{2}^{2}+\alpha_{3}^{2}=0$, where $\alpha_{1}, \alpha_{2}, \alpha_{3} \in \mathbb{F}$ in the former case and $\alpha_{1}, \alpha_{2}, \alpha_{3} \in \overline{\mathbb{F}}$ in the latter case. This shows that $\mathcal{D}_{\overline{\mathbb{F}}}(\chi) \neq\{o\}$ and that $\mathcal{D}(\chi)$ does not contain nonzero vectors of the form $\alpha_{1} e_{1}+\alpha_{2} e_{2}+\alpha_{3} e_{3}$.

We will now show that $\mathcal{D}(\chi)=\{o\}$. Suppose that this is not the case. Then there exists a vector $v_{1} \in \mathcal{D}(\chi) \backslash\left\langle e_{1}, e_{2}, e_{3}\right\rangle$. Let $v_{2}$ be a nonzero vector of $\left\langle e_{1}, e_{2}, e_{3}\right\rangle \cap v_{1}^{\perp_{f}}$ and let $v_{3}$ be a vector of $V$ such that $\left\langle v_{1}, v_{2}, v_{3}\right\rangle$ is a 3-dimensional totally isotropic subspace intersecting $\left\langle e_{1}, e_{2}, e_{3}\right\rangle$ in $\left\langle v_{2}\right\rangle$. Since $v_{1} \in \mathcal{D}(\chi)$, we have $\chi \wedge v_{1} \wedge v_{2} \wedge v_{3}=0$. Lemma 4.19 would now imply that $v_{2} \in \mathcal{D}(\chi)$, which is impossible as $v_{2} \in\left\langle e_{1}, e_{2}, e_{3}\right\rangle$.

Lemma 4.21 Let $\lambda_{1}, \lambda_{2} \in \mathbb{F}^{*}$ such that the equation $\lambda_{1} X^{2}+\lambda_{2} Y^{2}+Z^{2}=0$ has no solutions for $(X, Y, Z) \in \mathbb{F}^{3} \backslash\{(0,0,0)\}$. Then the following are equivalent for two linearly independent vectors $v_{1}, v_{2}$ of $V$ satisfying $f\left(v_{1}, v_{2}\right)=0$ :

(1) $v_{1}, v_{2} \in\left\langle e_{1}, e_{2}, e_{3}\right\rangle$;

(2) $\chi_{D 3}\left(\lambda_{1}, \lambda_{2}\right) \wedge v_{1} \wedge v_{2} \wedge v_{3}=0$ for every vector $v_{3} \in V$ such that $\left\langle v_{1}, v_{2}, v_{3}\right\rangle$ is a totally isotropic 3-space.

Proof. Clearly, (1) implies (2) (as $\chi_{D 3}\left(\lambda_{1}, \lambda_{2}\right) \wedge v_{1} \wedge v_{2}=0$ in that case). Conversely, suppose that (2) holds and that not both of $v_{1}, v_{2}$ are contained in $\left\langle e_{1}, e_{2}, e_{3}\right\rangle$. Then we can choose a vector $v_{3}$ such that $\left\langle v_{1}, v_{2}, v_{3}\right\rangle$ is a totally isotropic 3 -space intersecting 
$\left\langle e_{1}, e_{2}, e_{3}\right\rangle$ in a one-dimensional subspace $\langle e\rangle$. By Lemma 4.19, we would then have that $e \in \mathcal{D}\left(\chi_{D 3}\left(\lambda_{1}, \lambda_{2}\right)\right)$. But this is in contradiction with the fact that $\mathcal{D}\left(\chi_{D 3}\left(\lambda_{1}, \lambda_{2}\right)\right)=\{o\}$ (see Lemma 4.20).

Lemma 4.22 (1) The set $\mathcal{D}\left(\chi_{A 2}\right)$ is the union of all onedimensional subspaces of the form $\left\langle e_{1}\right\rangle,\left\langle b e_{2}+\beta f_{2}\right\rangle,\left\langle c e_{3}+\gamma f_{3}\right\rangle,\left\langle e_{1}+b e_{2}+\beta f_{2}\right\rangle,\left\langle e_{1}+c e_{3}+\gamma f_{3}\right\rangle$, where $(b, \beta)$ and $(c, \gamma)$ belong to $\mathbb{F}^{2} \backslash\{(0,0)\}$.

(2) For every $\lambda \in \mathbb{F}$ such that the polynomial $X^{2}+\lambda X+1 \in \mathbb{F}[X]$ is irreducible, we have $\mathcal{D}\left(\chi_{B 5}(\lambda)\right)=\left\langle e_{1}\right\rangle$.

(3) For every $\lambda \in \mathbb{F}^{*}$, we have $\mathcal{D}\left(\chi_{D 2}(\lambda)\right)=\mathcal{D}_{\overline{\mathbb{F}}}\left(\chi_{D 2}(\lambda)\right)=\{o\}$.

(4) Let $\lambda_{1}, \lambda_{2} \in \mathbb{F}^{*}$. Then $\mathcal{D}\left(\chi_{D 4}\left(\lambda_{1}, \lambda_{2}\right)\right)$ is either $\{o\}$ or the union of $|\mathbb{F}|+1$ onedimensional subspaces contained in $U:=\left\langle e_{1}, e_{2}, e_{3}\right\rangle$ defining a nonempty nonsingular conic of the projective plane $\mathrm{PG}(U)$, and $\mathcal{D}_{\overline{\mathbb{F}}}\left(\chi_{D 4}\left(\lambda_{1}, \lambda_{2}\right)\right)$ is the union of $|\overline{\mathbb{F}}|+1$ onedimensional subspaces contained in $\widetilde{U}:=\left\langle e_{1}, e_{2}, e_{3}\right\rangle_{\overline{\mathbb{F}}}$, defining a nonempty nonsingular conic of $\mathrm{PG}(\widetilde{U})$.

Proof. In [17], a method was described to determine the sets $\mathcal{D}(\chi)$ for trivectors $\chi$ of $V$. In Section 3 of that paper, this method was applied to determine these sets for some particular trivectors. Claims (1), (2) and (3) of the lemma follow from that treatment. We will now also prove Claim (4).

Put $\chi:=\chi_{D 4}\left(\lambda_{1}, \lambda_{2}\right)$ and let $\mathbb{K} \in\{\mathbb{F}, \overline{\mathbb{F}}\}$. The elements of $\mathcal{D}_{\mathbb{K}}(\chi)$ of the form $\alpha_{1} e_{1}+$ $\alpha_{2} e_{2}+\alpha_{3} e_{3}$ are determined by the equation $\lambda_{1} \alpha_{1}^{2}+\lambda_{1} \alpha_{1} \alpha_{3}+\alpha_{3}^{2}+\lambda_{2} \alpha_{2}^{2}=0$ where $\alpha_{1}, \alpha_{2}, \alpha_{3} \in$ $\mathbb{K}$. This equation determines a nonsingular conic of $\operatorname{PG}\left(\left\langle e_{1}, e_{2}, e_{3}\right\rangle_{\mathbb{K}}\right)$. If this conic is nonempty (which is always the case if $\mathbb{K}=\overline{\mathbb{F}}$ ), then it contains precisely $|\mathbb{K}|+1$ points. Observe that regardless of whether $\mathbb{K}=\mathbb{F}$ or $\mathbb{K}=\overline{\mathbb{F}}$, the conic can never contain a line of $\mathrm{PG}\left(\left\langle e_{1}, e_{2}, e_{3}\right\rangle_{\mathbb{K}}\right)$. In view of what we need to prove, it now suffices to show that the set $\mathcal{D}_{\mathbb{K}}(\chi) \backslash\left\langle e_{1}, e_{2}, e_{3}\right\rangle_{\mathbb{K}}$ is empty. Suppose that this is not the case and let $v_{1}$ be a vector belonging to this set. Then we will show that every nonzero vector $v_{2}$ belonging to the 2-space $v_{1}^{\perp_{\mathbb{K}}} \cap\left\langle e_{1}, e_{2}, e_{3}\right\rangle_{\mathbb{K}}$ belongs to $\mathcal{D}_{\mathbb{K}}(\chi)$, which is in contradiction with the abovementioned fact that the conic does not contain lines. Having chosen such a $v_{2}$, we choose a vector $v_{3} \in V_{\mathbb{K}}$ such that $\left\langle v_{1}, v_{2}, v_{3}\right\rangle_{\mathbb{K}}$ is a 3 -dimensional subspace, totally isotropic for $f_{\mathbb{K}}$, intersecting $\left\langle e_{1}, e_{2}, e_{3}\right\rangle_{\mathbb{K}}$ in $\left\langle v_{2}\right\rangle_{\mathbb{K}}$. Since $v_{1} \in \mathcal{D}_{\mathbb{K}}(\chi)$, we have $\chi \wedge v_{1} \wedge v_{2} \wedge v_{3}=0$. Lemma 4.19 would now imply that $v_{2} \in \mathcal{D}_{\mathbb{K}}(\chi)$. As this holds for every $v_{2} \in v_{1}^{\perp_{f_{K}}} \cap\left\langle e_{1}, e_{2}, e_{3}\right\rangle_{\mathbb{K}}$, we have obtained our desired contradiction.

Lemma 4.23 Let $i, j \in\{1,2, \ldots, 9\}$ with $i \neq j$. Then no trivector of Type $\left(Q i^{\prime}\right)$ is quasi-Sp $(V, f)$-equivalent with a trivector of Type $\left(Q j^{\prime}\right)$.

Proof. This is a consequence of Corollary 4.17 and Lemmas 3.9, 4.18, 4.20, 4.22, also taking into account that if two trivectors of $V$ are $S p(V, f)$-equivalent, then they are also $S p\left(V_{\overline{\mathbb{F}}}, f_{\overline{\mathbb{F}}}\right)$-equivalent. 
So, it remains to study the quasi- $S p(V, f)$-equivalence between trivectors having the same type $\left(\mathrm{Q} i^{\prime}\right)$, where $i \in\{4,5,6,7,8,9\}$. This will be done in the next six lemmas. These six lemmas correspond to the six claims of Theorem 1.5 that still need to be proved.

Lemma 4.24 Let $\lambda$ and $\lambda^{\prime}$ be two nonsquares of $\mathbb{F}$. Then the trivectors $\chi_{B 4}(\lambda)$ and $\chi_{B 4}\left(\lambda^{\prime}\right)$ are quasi-Sp $(V, f)$-equivalent if and only if the polynomials $X^{2}+\lambda$ and $X^{2}+\lambda^{\prime}$ define the same quadratic extension of $\mathbb{F}$ in $\overline{\mathbb{F}}$.

Proof. Let $\mathbb{K}=\mathbb{F}(\sqrt{\lambda}) \subseteq \overline{\mathbb{F}}$ and $\mathbb{K}^{\prime}=\mathbb{F}\left(\sqrt{\lambda^{\prime}}\right) \subseteq \overline{\mathbb{F}}$ be the quadratic extensions of $\mathbb{F}$ defined by the respective irreducible quadratic polynomials $X^{2}+\lambda$ and $X^{2}+\lambda^{\prime}$ of $\mathbb{F}[X]$.

Suppose the trivectors $\chi_{B 4}(\lambda)$ and $\chi_{B 4}\left(\lambda^{\prime}\right)$ are quasi-Sp $(V, f)$-equivalent. Then they are also quasi- $S p\left(V_{\mathbb{K}}, f_{\mathbb{K}}\right)$-equivalent. By Lemma $4.12, \chi_{B 4}(\lambda)$ is quasi- $S p\left(V_{\mathbb{K}}, f_{\mathbb{K}}\right)$-equivalent with $\chi_{A 1}$ and hence also $\chi_{B 4}\left(\lambda^{\prime}\right)$ should be quasi- $S p\left(V_{\mathbb{K}}, f_{\mathbb{K}}\right)$-equivalent with $\chi_{A 1}$. This implies by Lemma 3.9 and Lemma 4.20(1)+(2) that $\lambda^{\prime}$ should have a root in $\mathbb{K}$, i.e. $\mathbb{K}^{\prime}=\mathbb{F}\left(\sqrt{\lambda^{\prime}}\right) \subseteq \mathbb{K}$. By symmetry, we also have $\mathbb{K} \subseteq \mathbb{K}^{\prime}$. So, $\mathbb{K}=\mathbb{K}^{\prime}$.

Conversely, suppose that $\mathbb{K}=\mathbb{K}^{\prime}$. Then there exist $a, b \in \mathbb{F}$ with $a \neq 0$ such that $\lambda^{\prime}=a^{2} \lambda+b^{2}$. The element of $S p(V, f)$ mapping the hyperbolic basis $\left(e_{1}, f_{1}, e_{2}, f_{2}, e_{3}, f_{3}\right)$ of $(V, f)$ to the hyperbolic basis $\left(a e_{1}, \frac{f_{1}}{a}, \frac{1}{a}\left(e_{2}+b f_{3}\right), a f_{2}, e_{3}+b f_{2}, f_{3}\right)$ of $(V, f)$ maps $\chi_{B 4}(\lambda)=$ $e_{1} \wedge e_{2} \wedge e_{3}+\lambda \cdot e_{1} \wedge f_{2} \wedge f_{3}$ to the trivector

$$
e_{1} \wedge e_{2} \wedge e_{3}+\left(a^{2} \lambda+b^{2}\right) \cdot e_{1} \wedge f_{2} \wedge f_{3}+\left(b e_{1}\right) \wedge\left(e_{2} \wedge f_{2}+e_{3} \wedge f_{3}\right)=\chi_{B 4}\left(\lambda^{\prime}\right)+\phi\left(b e_{1}\right) .
$$

We conclude that $\chi_{B 4}(\lambda)$ and $\chi_{B 4}\left(\lambda^{\prime}\right)$ are quasi- $S p(V, f)$-equivalent.

Lemma 4.25 Let $\lambda, \lambda^{\prime} \in \mathbb{F}$ such that the polynomials $X^{2}+\lambda X+1$ and $X^{2}+\lambda^{\prime} X+1$ of $\mathbb{F}[X]$ are irreducible. Then the trivectors $\chi_{B 5}(\lambda)$ and $\chi_{B 5}\left(\lambda^{\prime}\right)$ are quasi-Sp $(V, f)$-equivalent if and only if the polynomials $X^{2}+\lambda X+1$ and $X^{2}+\lambda^{\prime} X+1$ define the same quadratic extension of $\mathbb{F}$ in $\overline{\mathbb{F}}$.

Proof. Let $\mathbb{K} \subseteq \overline{\mathbb{F}}$ and $\mathbb{K}^{\prime} \subseteq \overline{\mathbb{F}}$ be the quadratic extensions of $\mathbb{F}$ defined by the respective irreducible quadratic polynomials $X^{2}+\lambda X+1$ and $X^{2}+\lambda^{\prime} X+1$.

Suppose the trivectors $\chi_{B 5}(\lambda)$ and $\chi_{B 5}\left(\lambda^{\prime}\right)$ are quasi- $S p(V, f)$-equivalent. Then they are also quasi- $S p\left(V_{\mathbb{K}}, f_{\mathbb{K}}\right)$-equivalent. By Lemma $4.13, \chi_{B 5}(\lambda)$ is quasi- $S p\left(V_{\mathbb{K}}, f_{\mathbb{K}}\right)$-equivalent with $\chi_{A 2}$ and hence also $\chi_{B 5}\left(\lambda^{\prime}\right)$ should be quasi- $S p\left(V_{\mathbb{K}}, f_{\mathbb{K}}\right)$-equivalent with $\chi_{A 2}$. This implies by Lemma 3.9 and Lemma $4.22(1)+(2)$ that the quadratic polynomial $X^{2}+\lambda^{\prime} X+1$ should have its roots in $\mathbb{K}$. Hence, $\mathbb{K}^{\prime} \subseteq \mathbb{K}$. By symmetry, we also have $\mathbb{K} \subseteq \mathbb{K}^{\prime}$. So, $\mathbb{K}=\mathbb{K}^{\prime}$.

Conversely, suppose that $\mathbb{K}=\mathbb{K}^{\prime}$. Then there exist $a, b \in \mathbb{F}$ with $a \neq 0$ such that $\lambda^{\prime}=a \lambda$ and $a^{2}+b^{2}+a b \lambda=1$ (or equivalently, $(a X+b)^{2}+\lambda^{\prime}(a X+b)+1=a^{2}\left(X^{2}+\lambda X+1\right)$ ). We need to prove that the trivectors $\chi_{B 5}(\lambda)$ and $\chi_{B 5}\left(\lambda^{\prime}\right)$ are quasi- $S p(V, f)$-equivalent, or equivalently, that the trivectors $\chi:=\lambda \cdot e_{1} \wedge e_{2} \wedge f_{2}+e_{1} \wedge e_{2} \wedge f_{3}+e_{1} \wedge e_{3} \wedge f_{2}$ and $\chi^{\prime}:=\lambda^{\prime} \cdot e_{1} \wedge e_{2} \wedge f_{2}+e_{1} \wedge e_{2} \wedge f_{3}+e_{1} \wedge e_{3} \wedge f_{2}$ are quasi- $S p(V, f)$-equivalent. Now, let $\theta$ be the element of $S p(V, f)$ mapping the hyperbolic basis $\left(e_{1}, f_{1}, e_{2}, f_{2}, e_{3}, f_{3}\right)$ of $(V, f)$ 
to the hyperbolic basis $\left(a e_{1}, \frac{f_{1}}{a}, e_{2}+b e_{3}, f_{2}, a e_{3}, \frac{b f_{2}+f_{3}}{a}\right)$ of $(V, f)$. Then $\theta$ maps $\chi$ to the trivector

$$
(\lambda a) \cdot e_{1} \wedge e_{2} \wedge f_{2}+e_{1} \wedge e_{2} \wedge f_{3}+e_{1} \wedge e_{3} \wedge f_{2}+b e_{1} \wedge\left(e_{2} \wedge f_{2}+e_{3} \wedge f_{3}\right)=\chi^{\prime}+\phi\left(b e_{1}\right) .
$$

It follows that the trivectors $\chi$ and $\chi^{\prime}$ are quasi- $S p(V, f)$-equivalent and hence also the trivectors $\chi_{B 5}(\lambda)$ and $\chi_{B 5}\left(\lambda^{\prime}\right)$.

Lemma 4.26 Let $\lambda, \lambda^{\prime} \in \mathbb{F}^{*}$. Then $\chi_{C 1}(\lambda)$ and $\chi_{C 1}\left(\lambda^{\prime}\right)$ are quasi-Sp $(V, f)$-equivalent if and only if $\lambda=\lambda^{\prime}$.

Proof. If $\chi_{C 1}(\lambda)$ and $\chi_{C 1}\left(\lambda^{\prime}\right)$ are quasi-Sp $(V, f)$-equivalent, then $\lambda=\eta\left(\chi_{C 1}(\lambda)\right)=$ $\eta\left(\chi_{C 1}\left(\lambda^{\prime}\right)\right)=\lambda^{\prime}$ by Corollary 3.13.

Lemma 4.27 Let $\lambda, \lambda^{\prime} \in \mathbb{F}^{*}$. Then $\chi_{D 2}(\lambda)$ and $\chi_{D 2}\left(\lambda^{\prime}\right)$ are quasi-Sp $(V, f)$-equivalent if and only if $\lambda=\lambda^{\prime}$.

Proof. Suppose the trivectors $\chi_{D 2}(\lambda)$ and $\chi_{D 2}\left(\lambda^{\prime}\right)$ are quasi- $S p(V, f)$-equivalent. Then there exists a hyperbolic basis $\left(e_{1}^{\prime}, f_{1}^{\prime}, e_{2}^{\prime}, f_{2}^{\prime}, e_{3}^{\prime}, f_{3}^{\prime}\right)$ of $(V, f)$ and a $v \in V$ such that

$$
\begin{aligned}
& \lambda^{\prime} \cdot e_{1} \wedge e_{2} \wedge f_{3}+e_{2} \wedge f_{1} \wedge e_{3}+f_{1} \wedge e_{1} \wedge f_{2}+\phi(v) \\
= & \lambda \cdot e_{1}^{\prime} \wedge e_{2}^{\prime} \wedge f_{3}^{\prime}+e_{2}^{\prime} \wedge f_{1}^{\prime} \wedge e_{3}^{\prime}+f_{1}^{\prime} \wedge e_{1}^{\prime} \wedge f_{2}^{\prime} .
\end{aligned}
$$

If we let the map $\pi$ act on both sides of the equality (1), then we find $f_{2}=f_{2}^{\prime}$. Now, put $v=a_{1} e_{1}+a_{2} e_{2}+a_{3} e_{3}+b_{1} f_{1}+b_{2} f_{2}+b_{3} f_{3}$ where $a_{1}, a_{2}, a_{3}, b_{1}, b_{2}, b_{3} \in \mathbb{F}$. Then $\phi(v)$ is equal to

$$
\begin{aligned}
& a_{1} \cdot e_{1} \wedge\left(e_{2} \wedge f_{2}+e_{3} \wedge f_{3}\right)+a_{2} \cdot e_{2} \wedge\left(e_{1} \wedge f_{1}+e_{3} \wedge f_{3}\right)+a_{3} \cdot e_{3} \wedge\left(e_{1} \wedge f_{1}+e_{2} \wedge f_{2}\right) \\
& +b_{1} \cdot f_{1} \wedge\left(e_{2} \wedge f_{2}+e_{3} \wedge f_{3}\right)+b_{2} \cdot f_{2} \wedge\left(e_{1} \wedge f_{1}+e_{3} \wedge f_{3}\right)+b_{3} \cdot f_{3} \wedge\left(e_{1} \wedge f_{1}+e_{2} \wedge f_{2}\right)
\end{aligned}
$$

So, if we let the map $\eta$ act on both sides of the equality (1), then we find that $a_{2}=0$. Now, take the wedge product of both sides of (1) with $f_{2}=f_{2}^{\prime}$. Then we find

$$
\begin{aligned}
& \lambda^{\prime} \cdot e_{1} \wedge e_{2} \wedge f_{3} \wedge f_{2}+e_{2} \wedge f_{1} \wedge e_{3} \wedge f_{2}+a_{1} \cdot e_{1} \wedge e_{3} \wedge f_{3} \wedge f_{2}+a_{3} \cdot e_{3} \wedge e_{1} \wedge f_{1} \wedge f_{2}+ \\
& b_{1} \cdot f_{1} \wedge e_{3} \wedge f_{3} \wedge f_{2}+b_{3} \cdot f_{3} \wedge e_{1} \wedge f_{1} \wedge f_{2}=\lambda \cdot e_{1}^{\prime} \wedge e_{2}^{\prime} \wedge f_{3}^{\prime} \wedge f_{2}^{\prime}+e_{2}^{\prime} \wedge f_{1}^{\prime} \wedge e_{3}^{\prime} \wedge f_{2}^{\prime} .
\end{aligned}
$$

If we let $\pi^{\prime}$ act on both sides of the latter equality, then we find

$$
\begin{aligned}
& \lambda^{\prime} \cdot e_{1} \wedge f_{3}+f_{1} \wedge e_{3}+a_{1} \cdot e_{1} \wedge f_{2}+a_{3} \cdot e_{3} \wedge f_{2}+b_{1} \cdot f_{1} \wedge f_{2}+b_{3} \cdot f_{3} \wedge f_{2} \\
= & \lambda \cdot e_{1}^{\prime} \wedge f_{3}^{\prime}+f_{1}^{\prime} \wedge e_{3}^{\prime} .
\end{aligned}
$$

Since $\left(f_{2}^{\prime}\right)^{\perp_{f}}=f_{2}^{\perp_{f}}=\left\langle e_{1}, e_{3}, f_{1}, f_{3}, f_{2}\right\rangle=\left\langle e_{1}^{\prime}, e_{3}^{\prime}, f_{1}^{\prime}, f_{3}^{\prime}, f_{2}^{\prime}\right\rangle$, there exist unique vectors $e_{1}^{\prime \prime}, e_{3}^{\prime \prime}, f_{1}^{\prime \prime}, f_{3}^{\prime \prime} \in U:=\left\langle e_{1}, e_{3}, f_{1}, f_{3}\right\rangle$ and unique $\alpha, \beta, \gamma, \delta \in \mathbb{F}$ such that $e_{1}^{\prime}=e_{1}^{\prime \prime}+\alpha f_{2}$, $e_{3}^{\prime}=e_{3}^{\prime \prime}+\beta f_{2}, f_{1}^{\prime}=f_{1}^{\prime \prime}+\gamma f_{2}$ and $f_{3}^{\prime}=f_{3}^{\prime \prime}+\delta f_{2}$. Since $f\left(e_{1}^{\prime}, e_{3}^{\prime}\right)=f\left(e_{1}^{\prime}, f_{3}^{\prime}\right)=f\left(f_{1}^{\prime}, e_{3}^{\prime}\right)=$ $f\left(f_{1}^{\prime}, f_{3}^{\prime}\right)=0$ and $f\left(e_{1}^{\prime}, f_{1}^{\prime}\right)=f\left(e_{3}^{\prime}, f_{3}^{\prime}\right)=1$, also $f\left(e_{1}^{\prime \prime}, e_{3}^{\prime \prime}\right)=f\left(e_{1}^{\prime \prime}, f_{3}^{\prime \prime}\right)=f\left(f_{1}^{\prime \prime}, e_{3}^{\prime \prime}\right)=$ 
$f\left(f_{1}^{\prime \prime}, f_{3}^{\prime \prime}\right)=0$ and $f\left(e_{1}^{\prime \prime}, f_{1}^{\prime \prime}\right)=f\left(e_{3}^{\prime \prime}, f_{3}^{\prime \prime}\right)=1$. So, both $\left(e_{1}^{\prime \prime}, f_{1}^{\prime \prime}, e_{3}^{\prime \prime}, f_{3}^{\prime \prime}\right)$ and $\left(e_{1}, f_{1}, e_{3}, f_{3}\right)$ are hyperbolic bases of $\left(U, f_{\mid U}\right)$. Now, equation (2) becomes

$$
\begin{gathered}
\lambda^{\prime} \cdot e_{1} \wedge f_{3}+f_{1} \wedge e_{3}+a_{1} \cdot e_{1} \wedge f_{2}+a_{3} \cdot e_{3} \wedge f_{2}+b_{1} \cdot f_{1} \wedge f_{2}+b_{3} \cdot f_{3} \wedge f_{2} \\
=\lambda \cdot e_{1}^{\prime \prime} \wedge f_{3}^{\prime \prime}+f_{1}^{\prime \prime} \wedge e_{3}^{\prime \prime}+(\lambda \delta) \cdot e_{1}^{\prime \prime} \wedge f_{2}+(\lambda \alpha) \cdot f_{2} \wedge f_{3}^{\prime \prime}+\beta \cdot f_{1}^{\prime \prime} \wedge f_{2}+\gamma \cdot f_{2} \wedge e_{3}^{\prime \prime} .
\end{gathered}
$$

Since $e_{1}^{\prime \prime}, f_{1}^{\prime \prime}, e_{3}^{\prime \prime}, f_{3}^{\prime \prime} \in\left\langle e_{1}, f_{1}, e_{3}, f_{3}\right\rangle$, this implies that

$$
\lambda^{\prime} \cdot e_{1} \wedge f_{3}+f_{1} \wedge e_{3}=\lambda \cdot e_{1}^{\prime \prime} \wedge f_{3}^{\prime \prime}+f_{1}^{\prime \prime} \wedge e_{3}^{\prime \prime},
$$

and

$$
a_{1} e_{1}+a_{3} e_{3}+b_{1} f_{1}+b_{3} f_{3}=(\lambda \delta) e_{1}^{\prime \prime}+\beta f_{1}^{\prime \prime}+\gamma e_{3}^{\prime \prime}+(\lambda \alpha) f_{3}^{\prime \prime} .
$$

By Lemma 3.1, the former equation implies that

$$
\lambda^{\prime} \cdot e_{1} \wedge f_{1} \wedge e_{3} \wedge f_{3}=\lambda \cdot e_{1}^{\prime \prime} \wedge f_{1}^{\prime \prime} \wedge e_{3}^{\prime \prime} \wedge f_{3}^{\prime \prime} .
$$

Since $\left(e_{1}, f_{1}, e_{3}, f_{3}\right)$ and $\left(e_{1}^{\prime \prime}, f_{1}^{\prime \prime}, e_{3}^{\prime \prime}, f_{3}^{\prime \prime}\right)$ are two hyperbolic bases of $\left(U, f_{\mid U}\right)$, we have $e_{1} \wedge$ $f_{1} \wedge e_{3} \wedge f_{3}=e_{1}^{\prime \prime} \wedge f_{1}^{\prime \prime} \wedge e_{3}^{\prime \prime} \wedge f_{3}^{\prime \prime}\left(\right.$ Cohn [6, Corollary 3.6.4]). We conclude that $\lambda^{\prime}=\lambda$.

Lemma 4.28 Let $\lambda_{1}, \lambda_{2}, \lambda_{1}^{\prime}, \lambda_{2}^{\prime} \in \mathbb{F}^{*}$ such that none of the equations $\lambda_{1} X^{2}+\lambda_{2} Y^{2}+Z^{2}=0$ and $\lambda_{1}^{\prime} X^{2}+\lambda_{2}^{\prime} Y^{2}+Z^{2}=0$ has solutions for $(X, Y, Z) \in \mathbb{F}^{3} \backslash\{(0,0,0)\}$. Then the trivectors $\chi_{D 3}\left(\lambda_{1}, \lambda_{2}\right)$ and $\chi_{D 3}\left(\lambda_{1}^{\prime}, \lambda_{2}^{\prime}\right)$ are quasi-Sp $(V, f)$-equivalent if and only if there exists a $\mu \in \mathbb{F}^{*}$ such that the matrices $\mu \cdot \operatorname{diag}\left(\lambda_{1}, \lambda_{2}, 1\right)$ and $\operatorname{diag}\left(\lambda_{1}^{\prime}, \lambda_{2}^{\prime}, 1\right)$ are pseudocongruent.

Proof. If the matrices $\mu \cdot \operatorname{diag}\left(\lambda_{1}, \lambda_{2}, 1\right)$ and $\operatorname{diag}\left(\lambda_{1}^{\prime}, \lambda_{2}^{\prime}, 1\right)$ are pseudo-congruent for some $\mu \in \mathbb{F}^{*}$, then Lemma 3.14 implies that the trivectors $\chi_{D 3}\left(\lambda_{1}, \lambda_{2}\right)$ and $\chi_{D 3}\left(\lambda_{1}^{\prime}, \lambda_{2}^{\prime}\right)$ are quasi- $S p(V, f)$-equivalent.

Conversely, suppose that the trivectors $\chi_{D 3}\left(\lambda_{1}, \lambda_{2}\right)$ and $\chi_{D 3}\left(\lambda_{1}^{\prime}, \lambda_{2}^{\prime}\right)$ are quasi- $S p(V, f)$ equivalent. Then there exists a hyperbolic basis $\left(e_{1}^{\prime}, f_{1}^{\prime}, e_{2}^{\prime}, f_{2}^{\prime}, e_{3}^{\prime}, f_{3}^{\prime}\right)$ of $(V, f)$ and a vector $v \in V$ such that

$$
\begin{aligned}
& e_{1} \wedge e_{2} \wedge f_{3}+\lambda_{1} \cdot e_{2} \wedge e_{3} \wedge f_{1}+\lambda_{2} \cdot e_{3} \wedge e_{1} \wedge f_{2}+\phi(v) \\
& =\chi:=e_{1}^{\prime} \wedge e_{2}^{\prime} \wedge f_{3}^{\prime}+\lambda_{1}^{\prime} \cdot e_{2}^{\prime} \wedge e_{3}^{\prime} \wedge f_{1}^{\prime}+\lambda_{2}^{\prime} \cdot e_{3}^{\prime} \wedge e_{1}^{\prime} \wedge f_{2}^{\prime} .
\end{aligned}
$$

By Lemma 4.21, there exists a (necessarily unique) totally isotropic 3 -space $U$ such that the following are equivalent for two linearly independent vectors $v_{1}$ and $v_{2}$ of $V$ for which $f\left(v_{1}, v_{2}\right)=0$ :

(1) $v_{1}, v_{2} \in U$

(2) $\chi \wedge v_{1} \wedge v_{2} \wedge v_{3}=\chi_{D 3}\left(\lambda_{1}, \lambda_{2}\right) \wedge v_{1} \wedge v_{2} \wedge v_{3}=0$ for every vector $v_{3} \in V$ such that $\left\langle v_{1}, v_{2}, v_{3}\right\rangle$ is a totally isotropic 3 -space. 
Lemma 4.21 implies moreover that $U=\left\langle e_{1}, e_{2}, e_{3}\right\rangle$ and $U=\left\langle e_{1}^{\prime}, e_{2}^{\prime}, e_{3}^{\prime}\right\rangle$. Hence, $\left\langle e_{1}, e_{2}, e_{3}\right\rangle=$ $\left\langle e_{1}^{\prime}, e_{2}^{\prime}, e_{3}^{\prime}\right\rangle$.

By Lemma 4.19, the set $\mathcal{D}_{\overline{\mathbb{F}}}\left(\chi_{D 3}\left(\lambda_{1}, \lambda_{2}\right)\right) \cap\left\langle e_{1}, e_{2}, e_{3}\right\rangle_{\overline{\mathbb{F}}}$ is the union of all onedimensional subspaces of the form $\left\langle\alpha e_{1}+\beta e_{2}+\gamma e_{3}\right\rangle$, where $(\alpha, \beta, \gamma) \neq(0,0,0)$ satisfies $\sqrt{\lambda_{1}} \cdot \alpha+\sqrt{\lambda_{2}} \cdot \beta+$ $\gamma=0$. Similarly, the set $\mathcal{D}_{\overline{\mathbb{F}}}(\chi) \cap\left\langle e_{1}, e_{2}, e_{3}\right\rangle_{\overline{\mathbb{F}}}$ is the union of all onedimensional subspaces of the form $\left\langle\alpha^{\prime} e_{1}^{\prime}+\beta^{\prime} e_{2}^{\prime}+\gamma^{\prime} e_{3}^{\prime}\right\rangle$, where $\left(\alpha^{\prime}, \beta^{\prime}, \gamma^{\prime}\right) \neq(0,0,0)$ satisfies $\sqrt{\lambda_{1}^{\prime}} \cdot \alpha^{\prime}+\sqrt{\lambda_{2}^{\prime}} \cdot \beta^{\prime}+\gamma^{\prime}=0$.

Now, since $\left\langle e_{1}, e_{2}, e_{3}\right\rangle=\left\langle e_{1}^{\prime}, e_{2}^{\prime}, e_{3}^{\prime}\right\rangle$, there exists a nonsingular $(3 \times 3)$-matrix $M$ over $\mathbb{F}$ such that $\left[e_{1}^{\prime}, e_{2}^{\prime}, e_{3}^{\prime}\right]^{T}=M \cdot\left[e_{1}, e_{2}, e_{3}\right]^{T}$. We have $\alpha e_{1}+\beta e_{2}+\gamma e_{3}=\alpha^{\prime} e_{1}^{\prime}+\beta^{\prime} e_{2}^{\prime}+\gamma^{\prime} e_{3}^{\prime}$, where $[\alpha, \beta, \gamma]=\left[\alpha^{\prime}, \beta^{\prime}, \gamma^{\prime}\right] \cdot M$. Moreover, if $[\alpha, \beta, \gamma]=\left[\alpha^{\prime}, \beta^{\prime}, \gamma^{\prime}\right] \cdot M$, then $[\alpha, \beta, \gamma]$. $\left[\sqrt{\lambda_{1}}, \sqrt{\lambda_{2}}, 1\right]^{T}=\left[\alpha^{\prime}, \beta^{\prime}, \gamma^{\prime}\right] \cdot M \cdot\left[\sqrt{\lambda_{1}}, \sqrt{\lambda_{2}}, 1\right]^{T}$.

Since $\mathcal{D}_{\overline{\mathbb{F}}}\left(\chi_{D 3}\left(\lambda_{1}, \lambda_{2}\right)\right)=\mathcal{D}_{\overline{\mathbb{F}}}(\chi)$, the equations $\left[\alpha^{\prime}, \beta^{\prime}, \gamma^{\prime}\right] \cdot M \cdot\left[\sqrt{\lambda_{1}}, \sqrt{\lambda_{2}}, 1\right]^{T}=0$ and $\left[\alpha^{\prime}, \beta^{\prime}, \gamma^{\prime}\right] \cdot\left[\sqrt{\lambda_{1}^{\prime}}, \sqrt{\lambda_{2}^{\prime}}, 1\right]^{T}=0$ should be equivalent. So, there should exist a $\mu \in \overline{\mathbb{F}} \backslash\{0\}$ such that $\left[\sqrt{\lambda_{1}^{\prime}}, \sqrt{\lambda_{2}^{\prime}}, 1\right]^{T}=\mu \cdot M \cdot\left[\sqrt{\lambda_{1}}, \sqrt{\lambda_{2}}, 1\right]^{T}$. Hence,

$$
\left\{\begin{array}{c}
\sqrt{\lambda_{1}^{\prime}}=\mu M_{11} \sqrt{\lambda_{1}}+\mu M_{12} \sqrt{\lambda_{2}}+\mu M_{13}, \\
\sqrt{\lambda_{2}^{\prime}}=\mu M_{21} \sqrt{\lambda_{1}}+\mu M_{22} \sqrt{\lambda_{2}}+\mu M_{23}, \\
1=\mu M_{31} \sqrt{\lambda_{1}}+\mu M_{32} \sqrt{\lambda_{2}}+\mu M_{33},
\end{array}\right.
$$

or equivalently,

$$
\left\{\begin{array}{c}
\lambda_{1}^{\prime}=\mu^{2} M_{11}^{2} \lambda_{1}+\mu^{2} M_{12}^{2} \lambda_{2}+\mu^{2} M_{13}^{2}, \\
\lambda_{2}^{\prime}=\mu^{2} M_{21}^{2} \lambda_{1}+\mu^{2} M_{22}^{2} \lambda_{2}+\mu^{2} M_{23}^{2}, \\
1=\mu^{2} M_{31}^{2} \lambda_{1}+\mu^{2} M_{32}^{2} \lambda_{2}+\mu^{2} M_{33}^{2} .
\end{array}\right.
$$

This implies that $\mu^{2} \in \mathbb{F}^{*}$ and that $\operatorname{diag}\left(\lambda_{1}^{\prime}, \lambda_{2}^{\prime}, 1\right)-\mu^{2} \cdot\left(M \cdot \operatorname{diag}\left(\lambda_{1}, \lambda_{2}, 1\right) \cdot M^{T}\right)$ is an alternate matrix. Hence, the matrices $\mu^{2} \cdot \operatorname{diag}\left(\lambda_{1}, \lambda_{2}, 1\right)$ and $\operatorname{diag}\left(\lambda_{1}^{\prime}, \lambda_{2}^{\prime}, 1\right)$ are pseudocongruent.

Lemma 4.29 Let $\lambda_{1}, \lambda_{2}, \lambda_{1}^{\prime}, \lambda_{2}^{\prime} \in \mathbb{F}^{*}$. Then the two trivectors $\chi_{D 4}\left(\lambda_{1}, \lambda_{2}\right)$ and $\chi_{D 4}\left(\lambda_{1}^{\prime}, \lambda_{2}^{\prime}\right)$ are quasi-Sp $(V, f)$-equivalent if and only if there exists a $\mu \in \mathbb{F}^{*}$ such that the matrices $\mu A$ and $A^{\prime}$ are pseudo-congruent, where

$$
A:=\left[\begin{array}{ccc}
\lambda_{1} & 0 & \lambda_{1} \\
0 & \lambda_{2} & 0 \\
0 & 0 & 1
\end{array}\right] \quad \text { and } \quad A^{\prime}:=\left[\begin{array}{ccc}
\lambda_{1}^{\prime} & 0 & \lambda_{1}^{\prime} \\
0 & \lambda_{2}^{\prime} & 0 \\
0 & 0 & 1
\end{array}\right]
$$

Proof. If the matrices $\mu A$ and $A^{\prime}$ are pseudo-congruent for some $\mu \in \mathbb{F}^{*}$, then Lemma 3.14 implies that the trivectors $\chi_{D 4}\left(\lambda_{1}, \lambda_{2}\right)$ and $\chi_{D 4}\left(\lambda_{1}^{\prime}, \lambda_{2}^{\prime}\right)$ are quasi- $S p(V, f)$-equivalent.

Conversely, suppose that the trivectors $\chi_{D 4}\left(\lambda_{1}, \lambda_{2}\right)$ and $\chi_{D 4}\left(\lambda_{1}^{\prime}, \lambda_{2}^{\prime}\right)$ are quasi- $S p(V, f)$ equivalent. Then there exists a hyperbolic basis $\left(e_{1}^{\prime}, f_{1}^{\prime}, e_{2}^{\prime}, f_{2}^{\prime}, e_{3}^{\prime}, f_{3}^{\prime}\right)$ of $(V, f)$ and a vector $v \in V$ such that

$$
\begin{aligned}
& e_{1} \wedge e_{2} \wedge f_{3}+\lambda_{1} \cdot e_{2} \wedge e_{3} \wedge\left(f_{1}+f_{3}\right)+\lambda_{2} \cdot e_{3} \wedge e_{1} \wedge f_{2}+\phi(v) \\
& =\chi:=e_{1}^{\prime} \wedge e_{2}^{\prime} \wedge f_{3}^{\prime}+\lambda_{1}^{\prime} \cdot e_{2}^{\prime} \wedge e_{3}^{\prime} \wedge\left(f_{1}^{\prime}+f_{3}^{\prime}\right)+\lambda_{2}^{\prime} \cdot e_{3}^{\prime} \wedge e_{1}^{\prime} \wedge f_{2}^{\prime} .
\end{aligned}
$$

By Lemma $4.22(4), \mathcal{D}_{\overline{\mathbb{F}}}\left(\chi_{D 4}\left(\lambda_{1}, \lambda_{2}\right)\right)=\mathcal{D}_{\overline{\mathbb{F}}}\left(\chi_{D 4}\left(\lambda_{1}, \lambda_{2}\right)+\phi(v)\right)$ generates the subspace $\left\langle e_{1}, e_{2}, e_{3}\right\rangle_{\overline{\mathbb{F}}}$, and $\mathcal{D}_{\overline{\mathbb{F}}}(\chi)$ generates the subspace $\left\langle e_{1}^{\prime}, e_{2}^{\prime}, e_{3}^{\prime}\right\rangle_{\overline{\mathbb{F}}}$. Hence, $\left\langle e_{1}, e_{2}, e_{3}\right\rangle=\left\langle e_{1}^{\prime}, e_{2}^{\prime}, e_{3}^{\prime}\right\rangle$. 
So, there exists a nonsingular $(3 \times 3)$-matrix $M$ over $\mathbb{F}$ such that $\left[e_{1}, e_{2}, e_{3}\right]^{T}=M$. $\left[e_{1}^{\prime}, e_{2}^{\prime}, e_{3}^{\prime}\right]^{T}$. Let $\alpha_{1} e_{1}+\alpha_{2} e_{2}+\alpha_{3} e_{3}$ be an arbitrary nonzero vector of $V_{\overline{\mathbb{F}}}$ contained in $\left\langle e_{1}, e_{2}, e_{3}\right\rangle_{\overline{\mathbb{F}}}$. We have $\alpha_{1} e_{1}+\alpha_{2} e_{2}+\alpha_{3} e_{3}=\alpha_{1}^{\prime} e_{1}^{\prime}+\alpha_{2}^{\prime} e_{2}^{\prime}+\alpha_{3}^{\prime} e_{3}^{\prime}$, where $\left[\alpha_{1}, \alpha_{2}, \alpha_{3}\right] \cdot M=$ $\left[\alpha_{1}^{\prime}, \alpha_{2}^{\prime}, \alpha_{3}^{\prime}\right]$. By Lemma 4.19, the onedimensional subspace $\left\langle\alpha_{1} e_{1}+\alpha_{2} e_{2}+\alpha_{3} e_{3}\right\rangle_{\overline{\mathrm{F}}}=\left\langle\alpha_{1}^{\prime} e_{1}^{\prime}+\right.$ $\left.\alpha_{2}^{\prime} e_{2}^{\prime}+\alpha_{3}^{\prime} e_{3}^{\prime}\right\rangle_{\overline{\mathbb{F}}}$ is contained in $\mathcal{D}_{\overline{\mathbb{F}}}\left(\chi_{D 4}\left(\lambda_{1}, \lambda_{2}\right)\right)=\mathcal{D}_{\overline{\mathbb{F}}}(\chi)$ if and only if one of the following (necessarily equivalent) conditions is satisfied:

- $\left[\alpha_{1}, \alpha_{2}, \alpha_{3}\right] \cdot A \cdot\left[\alpha_{1}, \alpha_{2}, \alpha_{3}\right]^{T}=0$,

- $\left[\alpha_{1}^{\prime}, \alpha_{2}^{\prime}, \alpha_{3}^{\prime}\right] \cdot A^{\prime} \cdot\left[\alpha_{1}^{\prime}, \alpha_{2}^{\prime}, \alpha_{3}^{\prime}\right]^{T}=0$.

Now, $\left[\alpha_{1}^{\prime}, \alpha_{2}^{\prime}, \alpha_{3}^{\prime}\right] \cdot A^{\prime} \cdot\left[\alpha_{1}^{\prime}, \alpha_{2}^{\prime}, \alpha_{3}^{\prime}\right]^{T}=\left[\alpha_{1}, \alpha_{2}, \alpha_{3}\right] \cdot\left(M A^{\prime} M^{T}\right) \cdot\left[\alpha_{1}, \alpha_{2}, \alpha_{3}\right]^{T}$. Since the equations $\left[\alpha_{1}, \alpha_{2}, \alpha_{3}\right] \cdot A \cdot\left[\alpha_{1}, \alpha_{2}, \alpha_{3}\right]^{T}=0$ and $\left[\alpha_{1}, \alpha_{2}, \alpha_{3}\right] \cdot\left(M A^{\prime} M^{T}\right) \cdot\left[\alpha_{1}, \alpha_{2}, \alpha_{3}\right]^{T}=0$ describe the same nondegenerate nonempty conic of $\mathrm{PG}\left(\left\langle e_{1}, e_{2}, e_{3}\right\rangle_{\overline{\mathbb{F}}}\right)$, there should exist a $\mu \in \mathbb{F}^{*}$ such that $\mu A-M A^{\prime} M^{T}$ is an alternate matrix. Hence, the matrices $\mu A$ and $A^{\prime}$ are pseudo-congruent.

Remark. We were also able to deal with some but not all of the trivectors of Type (E). Indeed, every trivector of Type (E2), (E3) or (E5) is quasi- $S p(V, f)$-equivalent with a trivector of Type $(\mathrm{X})$ with $(X) \in\{(B),(C),(D)\}$ Note that since $\operatorname{char}(\mathbb{F})=2$, we must have that $a \neq 0 \neq b$ for every $(a, b) \in \Psi$.

- Suppose $(a, b) \in \Psi$ and $k \in \mathbb{F}^{*}$. Then the trivector $\chi_{E 2}(a, b, k)$ of Type (E2) is quasi-Sp $(V, f)$-equivalent with $k \cdot\left(f_{1} \wedge e_{2} \wedge f_{3}+b \cdot f_{1} \wedge f_{2} \wedge e_{3}+a \cdot f_{1} \wedge e_{3} \wedge f_{3}\right)=$ $f_{1} \wedge f_{3} \wedge\left(k e_{2}+k a e_{3}\right)+f_{1} \wedge f_{2} \wedge\left(k b e_{3}\right)$ which is a trivector of Type (B).

- Suppose $(a, b) \in \Psi$ and $k, h \in \mathbb{F}^{*}$. Then the trivector $\chi_{E 3}(a, b, k, h)$ of Type (E3) is quasi- $S p(V, f)$-equivalent with $k \cdot\left(f_{1} \wedge e_{2} \wedge f_{3}+b \cdot f_{1} \wedge f_{2} \wedge e_{3}+a \cdot f_{1} \wedge e_{3} \wedge f_{3}\right)+h \cdot e_{1} \wedge f_{2} \wedge f_{3}=$ $f_{1} \wedge f_{3} \wedge\left(k e_{2}+k a e_{3}\right)+f_{1} \wedge f_{2} \wedge\left(k b e_{3}\right)+f_{2} \wedge f_{3} \wedge\left(h e_{1}\right)$ which is a trivector of Type (D).

- Suppose $(a, b) \in \Psi$ and $k \in \mathbb{F}^{*}$. Then the trivector $\chi_{E 5}(a, b, k)$ of Type (E5) is quasi$S p(V, f)$-equivalent with $f_{1} \wedge e_{2} \wedge f_{3}+a \cdot e_{2} \wedge f_{2} \wedge e_{3}+\left(a^{2}+b\right) \cdot f_{1} \wedge f_{2} \wedge e_{3}+k a \cdot e_{1} \wedge f_{2} \wedge e_{3}=$ $f_{1} \wedge e_{2} \wedge f_{3}+\left(a e_{2}+\left(a^{2}+b\right) f_{1}+k a e_{1}\right) \wedge f_{2} \wedge e_{3}$ which is a trivector of Type (C).

\section{References}

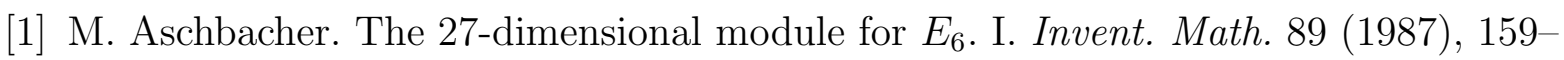
195.

[2] B. Capdevielle. Classification des formes trilinéaires alternées en dimension 6. Enseignement Math. 18 (1972), 225-243.

[3] A. M. Cohen and B. N. Cooperstein. The 2-spaces of the standard $E_{6}(q)$-module. Geometries and groups (Noordwijkerhout, 1986). Geom. Dedicata 25 (1988), 467480.

[4] A. M. Cohen and A. G. Helminck. Trilinear alternating forms on a vector space of dimension 7. Comm. Algebra 16 (1988), 1-25.

[5] A. M. Cohen and D. B. Wales. GL(4)-orbits in a 16-dimensional module for characteristic 3. J. Algebra 185 (1996), 85-107.

[6] P. M. Cohn. Further algebra and applications. Springer-Verlag London, London, 2003. 
[7] B. N. Cooperstein. The fifty-six-dimensional module for $E_{7}$. I. A four form for $E_{7} . J$. Algebra 173 (1995), 361-389.

[8] B. N. Cooperstein. On the generation of dual polar spaces of symplectic type over finite fields. J. Combin. Theory Ser. A 83 (1998), 221-232.

[9] B. N. Cooperstein and B. De Bruyn. Points and hyperplanes of the universal embedding space of the dual polar space $D W(5, q), q$ odd. Michigan Math. J. 58 (2009), $195-212$.

[10] B. De Bruyn. Hyperplanes of $D W(5, \mathbb{K})$ with $\mathbb{K}$ a perfect field of characteristic 2. J. Algebraic Combin. 30 (2009), 567-584.

[11] B. De Bruyn. Some subspaces of the $k$-th exterior power of a symplectic vector space. Linear Algebra Appl. 430 (2009), 3095-3104.

[12] B. De Bruyn. On polyvectors of vector spaces and hyperplanes of projective Grassmannians. Linear Algebra Appl. 435 (2011), 1055-1084.

[13] B. De Bruyn and M. Kwiatkowski. On the trivectors of a 6-dimensional symplectic vector space. Linear Algebra Appl. 435 (2011), 289-306.

[14] B. De Bruyn and M. Kwiatkowski. On the trivectors of a 6-dimensional symplectic vector space. II. Linear Algebra Appl. 437 (2012), 1215-1233.

[15] B. De Bruyn and M. Kwiatkowski. On the trivectors of a 6-dimensional symplectic vector space. III. Linear Algebra Appl. 438 (2013), 374-398.

[16] B. De Bruyn and M. Kwiatkowski. On the trivectors of a 6-dimensional symplectic vector space. IV. Linear Algebra Appl. 438 (2013), 2405-2429.

[17] B. De Bruyn and M. Kwiatkowski. On trivectors and hyperplanes of symplectic dual polar spaces: the deep quads. Preprint 2013.

[18] D. Ž. Djoković. Classification of trivectors of an eight-dimensional real vector space. Linear and Multilinear Algebra 13 (1983), 3-39.

[19] R. Gow. Contraction of exterior powers in characteristic 2 and the spin module. Geom. Dedicata 64 (1997), 283-295.

[20] R. M. Guralnick, M. W. Liebeck, D. Macpherson and G. M. Seitz. Modules for algebraic groups with finitely many orbits on subspaces. J. Algebra 196 (1997), 211250 .

[21] G. B. Gurevich. Sur les formes canoniques d'un trivecteur dans l'espace à six dimensions. C. R. Acad. Sci. (Paris) 197 (1933), 384.

[22] G. B. Gurevich. L'algèbre du trivecteur. Trudy Sem. Vektor. Tenzor. Anal., no. II-III (1935), 51-118.

[23] G. B. Gurevich. Classification des trivecteurs ayant le rang huit. Dokl. Akad. Nauk SSSR II, No. 5-6 (1935), 355-356.

[24] J.-i. Igusa. A classification of spinors up to dimension twelve. Amer. J. Math. 92 (1970), 997-1028.

[25] A. Kasikova and E. Shult. Absolute embeddings of point-line geometries. J. Algebra 238 (2001), 265-291. 
[26] V. L. Popov. Classification of spinors of dimension fourteen. Trans. Mosc. Math. Soc. 1 (1980), 181-232.

[27] A. A. Premet and I. D. Suprunenko. The Weyl modules and the irreducible representations of the symplectic group with the fundamental highest weights. Comm. Algebra 11 (1983), 1309-1342.

[28] W. Reichel. Über die trilinearen alternierenden Formen in 6 und 7 Veränderlichen. Dissertation, Greifswald, 1907.

[29] Ph. Revoy. Trivecteurs de rang 6. Colloque sur les Formes Quadratiques (Montpellier, 1977). Bull. Soc. Math. France Mém. 59 (1979), 141-155.

[30] Ph. Revoy. Formes trilinéaires alternées de rang 7. Bull. Sci. Math. 112 (1988), 357368.

[31] M. A. Ronan. Embeddings and hyperplanes of discrete geometries. European J. Combin. 8 (1987), 179-185.

[32] J. A. Schouten. Klassifizierung der alternierenden Grössen dritten Grades in 7 Dimensionen. Rend. Circ. Mat. Palermo 55 (1931), 137-156.

[33] È. B. Vinberg and A. G. Èlašvili. Classification of trivectors of a nine-dimensional space. Trudy Sem. Vektor. Tenzor. Anal. 18 (1978), 197-233.

[34] R. Westwick. Real trivectors of rank seven. Linear and Multilinear Algebra 10 (1981), 183-204. 